\title{
Cold gas accretion in galaxies
}

\author{
Renzo Sancisi - Filippo Fraternali • \\ Tom Oosterloo • Thijs van der Hulst
}

Received: 28 January 2008 / Published online: 17 April 2008

(C) The Author(s) 2008

\begin{abstract}
Evidence for the accretion of cold gas in galaxies has been rapidly accumulating in the past years. HI observations of galaxies and their environment have brought to light new facts and phenomena which are evidence of ongoing or recent accretion: (1) A large number of galaxies are accompanied by gas-rich dwarfs or are surrounded by H I cloud complexes, tails and filaments. This suggests ongoing minor mergers and recent arrival of external gas. It may be regarded, therefore, as direct evidence of cold gas accretion in the local universe. It is probably the same kind of phenomenon of material infall as the stellar streams observed in the halos of our galaxy and M31. (2) Considerable amounts of extra-planar Hi have been found in nearby spiral galaxies. While a large fraction of this gas is undoubtedly produced by galactic fountains, it is likely that a part of it is of extragalactic origin. Also the Milky Way has extra-planar gas complexes: the Intermediate- and High-Velocity Clouds (IVCs and HVCs). (3) Spirals are known to have extended and warped outer layers of HI. It is not clear how these have formed, and how and for how long the warps can
\end{abstract}

\footnotetext{
R. Sancisi $(\varangle)$

Osservatorio Astronomico di Bologna, Via Ranzani 1, 40127 Bologna, Italy

e-mail: sancisi@bo.astro.it
}

R. Sancisi - T. Oosterloo · T. van der Hulst

Kapteyn Astronomical Institute, Postbus 800, 9700 AV Groningen, The Netherlands

e-mail: sancisi@astro.rug.nl

T. van der Hulst

e-mail: vdhulst@astro.rug.nl

\section{F. Fraternali}

Astronomy Department, Bologna University, Via Ranzani 1, 40127 Bologna, Italy

e-mail: filippo.fraternali@unibo.it

T. Oosterloo

ASTRON, Postbus 2, 7990 AA Dwingeloo, The Netherlands

e-mail: oosterloo@astron.nl 
be sustained. Gas infall has been proposed as the origin. (4) The majority of galactic disks are lopsided in their morphology as well as in their kinematics. Also here recent accretion has been advocated as a possible cause. In our view, accretion takes place both through the arrival and merging of gas-rich satellites and through gas infall from the intergalactic medium (IGM). The new gas could be added to the halo or be deposited in the outer parts of galaxies and form reservoirs for replenishing the inner parts and feeding star formation. The infall may have observable effects on the disk such as bursts of star formation and lopsidedness. We infer a mean "visible" accretion rate of cold gas in galaxies of at least $0.2 \mathrm{M}_{\odot}$ year $^{-1}$. In order to reach the accretion rates needed to sustain the observed star formation $\left(\approx 1 \mathrm{M}_{\odot}\right.$ year $\left.^{-1}\right)$, additional infall of large amounts of gas from the IGM seems to be required.

Keywords Galaxies · Neutral hydrogen · Accretion · Extra-planar gas · Interactions · Mergers

\section{Introduction}

Gas accretion plays a fundamental role in the evolution of galaxies. Fresh supplies of gas are needed for the ongoing process of star formation. Such a process of galaxy "nurture" is expected to continue to the present day. The importance and role of gas infall for the evolution of disk galaxies have been recognized for many years (Larson 1972; Larson et al. 1980; Tinsley 1980; Tosi 1988).

The rate of star-formation in the solar neighborhood has been remarkably constant over the Milky Way's life (Twarog 1980; Binney et al. 2000), which suggests that the gas consumed by star formation has been replaced by accretion. Steady accretion of metal-poor gas would also explain the discrepancy between the observed stellar metallicity distribution in the solar neighborhood and that predicted by closed-box models of chemical evolution (Tinsley 1981; Matteucci 2003). It is not clear how much new gas is needed to sustain star formation. The star formation rate (SFR) varies throughout the Galactic disk and from galaxy to galaxy. Its value is still very uncertain. Here, we assume a reference global value of $1 \mathrm{M}_{\odot}$ year $^{-1}$ both for the average SFR and for the required gas accretion rate.

Several arguments suggest that most of the baryons in the local universe still reside in the intergalactic medium (White and Frenk 1991; Fukugita and Peebles 2004; Sommer-Larsen 2006). Out of this medium galaxies are expected to grow through a series of infall events ranging from a small number of major mergers down to an almost continuous infall of dwarf galaxies and gas clouds, the latter being more and more important at low redshifts (Bond et al. 1991; Lacey and Cole 1993). Recent, high-resolution cosmological simulations show that there are two modes of accretion: hot accretion, mainly around massive structures, and cold accretion (clouds, streams or filaments) for galaxies with lower halo masses, which correspond to the population of star forming galaxies (Dekel and Birnboim 2006). These arguments also point to a rate of gas accretion for galaxies which is very close to their star-formation rate (Kereš et al. 2005).

Direct observational evidence of accretion actually taking place has, however, been difficult to obtain. The study of H I in the Milky Way and in external galaxies has played 
a central role. For several decades H i observations have given valuable information on gaseous content, structure and kinematics of galaxies and on their environment. In particular, the $\mathrm{H}$ I rotation curves have provided the crucial evidence for dark matter in spiral galaxies. New facts have been revealed, especially in recent deep observations, which now constitute the best evidence for cold gas accretion. We briefly review them here.

First (Sect. 2), we focus on those phenomena-interactions, minor mergers, peculiar H I structures around galaxies - which in our opinion point directly at ongoing or recent processes of accretion. We believe that the stellar merger remnants recently discovered in the halo of our galaxy (e.g. Sgr Dwarf) (Ibata et al. 1994) and of M 31 (Ibata et al. 2001; Ferguson et al. 2002; McConnachie et al. 2003), and other faint optical features found around some nearby galaxies (Malin and Hadley 1997; Shang et al. 1998), are manifestations of the same phenomena as those revealed by H observations and described here.

Subsequently, we draw attention to other aspects of the structure and kinematics of $\mathrm{HI}$ in galaxies - the extra-planar gas, the extended and warped outer layers and the lopsidedness-which may be part of the accretion process. The connection between accretion and the above phenomena, however, is not entirely clear and most of the evidence is indirect. The extra-planar gas (Sect. 3), although in part undoubtedly a product of galactic fountains (Fraternali and Binney 2006), must also have a component which originated from infall or minor mergers (Oosterloo et al. 2007a; Fraternali and Binney 2008). In our discussion of extra-planar H I we include, together with the recent evidence from external galaxies, the long-known High-Velocity Clouds (HVCs), most of which are now conclusively regarded as a Milky Way halo population and direct evidence for infall of intergalactic gas (Wakker et al. 2007, 2008). The warped outer Hi layers (Sect. 4) of spirals may also be the result of infall and form a source of continuous supply of fresh gas for the inner disks. Finally (Sect. 5), also the lopsided $\mathrm{HI}$ morphology and kinematics and the asymmetric optical images of a large number of disks may have originated from recent minor mergers or large-scale cosmological gas accretion (Bournaud et al. 2005). The question of the intergalactic origin of the infalling gas is addressed in Sect. 6.

\section{Interactions and minor mergers}

\subsection{Dwarf companions and peculiar structures}

There are several cases of multiple systems with similar mass [e.g. M81-M82NGC 3077 (Yun et al. 1994), NGC4631-4656-4627 (Rand 1994), NGC5194 (M51)-5195 (Rots et al. 1990)] which show heavily disturbed H I images with associated cloud complexes, long tails, bridges and ring-like structures. For many of these systems, it is the peculiar Hi picture that unmistakably points at the ongoing strong tidal interactions and major mergers.

Here, however, we draw attention to galaxies which are interacting with gas-rich dwarf companions and to galaxies with no apparent interaction but with peculiar $\mathrm{HI}$ structures and/or kinematics. All these systems can be considered as minor mergers 
at different stages. Those with companions show H I tails and bridges indicating that an interaction is indeed taking place. The others, with no visible companions, have peculiar features in their H I structure and kinematics, especially in their outer parts, which are reminiscent of interacting systems. They may, therefore, have had some recent encounter and may be in an advanced stage of merging (Sancisi 1999a,b). However, as far as we know, they may also be the result of the infall of intergalactic gas clouds.

A number of representative cases are listed in Table 1. This list is by no means complete. A compilation of a large number of Hi maps of peculiar galaxies, which includes many cases of the kind discussed here, is provided by "An H i Rogues Gallery" (Hibbard et al. 2001).

Prototypes of galaxies with interacting dwarf companions are NGC 3359 (Fig. 1), NGC 4565-4565A (Fig. 4) and NGC 4027-4027A. The companions have systemic velocities close to those of the main galaxy and H I masses less than $10 \%$ of the main galaxy. The Hi picture suggests the capture of a gas-rich dwarf by a massive system, probably to be followed by tidal disruption and accretion of the dwarf. This would bring in gas, stars and dark matter.

The Milky Way and the Magellanic Clouds are in this class of phenomena and the Magellanic Stream (Mathewson et al. 1974; Brüns et al. 2005) is the gas component (about $1.2 \times 10^{8} \mathrm{M}_{\odot}$ ) probably destined to be accreted by our galaxy. The H I masses of LMC and SMC (4.4 and $4.0 \times 10^{8} \mathrm{M}_{\odot}$, respectively) are of the same order as those of the companions discussed here.

The cases just mentioned probably represent early stages of the interaction-accretion process. At later, more advanced stages, the victim may be no longer visible or not easy to be identified unambiguously. Examples are M 101 (see Sect. 2.2.1, Fig. 2), NGC 210 (see Sect. 2.2.2, Fig. 1), NGC 1023, NGC 3310, NGC628 and Mkn 348 (Fig. 1). NGC 1023 is an S0 galaxy surrounded by a clumpy and irregular H I structure of $1.0 \times 10^{9} \mathrm{M}_{\odot}$ (Sancisi et al. 1984), reminiscent of the tails and bridges found in interacting multiple systems. There are a few dwarf neighbors, one in particular on its eastern edge, which might be merger relics. NGC 3310 is a peculiar (Arp 217) Sbc starburst galaxy. Mulder et al. (1995) and Kregel and Sancisi (2001) have shown the presence of extended H I emission, which has a well developed two-tail structure. This must be an advanced merger that has either preserved the old disk of one of the progenitors or, perhaps more likely, has led to the formation of a new disk. Although the optical images of both NGC 1023 and NGC 3310, as of several other objects in Table 1 (e.g. M 101, NGC 925), already show some peculiarities, it is their HI structure and kinematics that fully reveal the ongoing mergers. Other galaxies, such as NGC 210, NGC 628 and Mkn 348, have a clean, regular optical image and only the H I betrays a possible recent accretion. For NGC 628 this is indicated by the presence in its outer parts of two giant high-velocity H I complexes, which are symmetrically placed with respect to the galaxy center. These complexes have H I masses of about $10^{8} \mathrm{M}_{\odot}$, and maximum velocity excesses of $100 \mathrm{~km} \mathrm{~s}^{-1}$ (Kamphuis and Briggs 1992). For Mkn 348 (NGC 262) a probable past interaction and gas accretion is suggested by the presence of an enormous Hi envelope [176 kpc diameter (Heckman et al. 1982)] and a large tail-like extension (Fig. 1) (Simkin et al. 1987). In all cases, like these latter, where there is no optical victim visible, we may be dealing with the accretion of a 
Table 1 Galaxies with dwarf companions and/or peculiar HI structures

\begin{tabular}{|c|c|c|c|}
\hline Object & Features & $\begin{array}{l}\text { Masses } \\
\left(10^{8} \mathrm{M}_{\odot}\right)\end{array}$ & References \\
\hline IC10 & - & - & Hibbard et al. (2001) \\
\hline NGC 210 & Tail & 6 & $\mathrm{a}$ \\
\hline NGC 262 (Mkn 348) & Tail & $>20$ & $\begin{array}{l}\text { Heckman et al. (1982), } \\
\text { Simkin et al. (1987) }\end{array}$ \\
\hline NGC 628 & High-velocity complexes & 2 & Kamphuis and Briggs (1992) \\
\hline NGC 925 & Tail & 3 & $\mathrm{~b}$ \\
\hline NGC 1023 (Arp 135) & Tail/ring & 10 & Sancisi et al. (1984) \\
\hline NGC 1961 (Arp 184) & Wing & $\sim 54$ & Shostak et al. (1982) \\
\hline NGC 2146 & Cloud & 46 & $\begin{array}{l}\text { Fisher and Tully (1976), } \\
\text { Taramopoulos et al. (2001) }\end{array}$ \\
\hline NGC 2782 (Arp 215) & Plume & $\sim 10$ & Smith (1994) \\
\hline NGC 2985 (UGC 5253) & Tail, interaction & $>3.6$ & $\mathrm{~b}$ \\
\hline NGC 3067 & Plume & 2 & Carilli and van Gorkom (1992) \\
\hline NGC 3310 (Arp 217) & Tails & 5 & $\begin{array}{l}\text { Mulder et al. (1995), } \\
\text { Kregel and Sancisi (2001) }\end{array}$ \\
\hline NGC 3359 & Companion/bridge & 9.6 & $\begin{array}{l}\text { Kamphuis and Sancisi (1993), } \\
\text { van der Hulst and Sancisi (2005) }\end{array}$ \\
\hline NGC 4027 (Arp 22) & Companion/ring & 6.6 & Phookun et al. (1992) \\
\hline NGC 4565 & Companions/bridge & $\sim 1$ & Rupen (1991) \\
\hline NGC 4826 & Counter-rotating disk & A few & Braun et al. (1994) \\
\hline NGC 5457 (M 101, Arp 26) & High-velocity complexes & 2 & $\begin{array}{l}\text { van der Hulst and Sancisi (1988), } \\
\text { Kamphuis (1993) }\end{array}$ \\
\hline NGC 5635 & Cloud & 2 & Saglia and Sancisi (1988) \\
\hline NGC 6946 & Plume & $\gtrsim 1$ & Boomsma et al. (2005b) \\
\hline Milky Way & Magellanic stream & 1.2 & $\begin{array}{l}\text { Mathewson et al. (1974), } \\
\text { Brüns et al. (2005) }\end{array}$ \\
\hline NGC 2865 & & & Schiminovich et al. (1995) \\
\hline NGC 3656 (Arp 155) & & & Balcells and Sancisi (1996) \\
\hline NGC 4472 (Arp 134) & & & McNamara et al. (1994) \\
\hline NGC 5128 (Cen A) & & & Schiminovich et al. (1994) \\
\hline
\end{tabular}

${ }^{\mathrm{a}}$ G. Gentile, private communication

b $\mathrm{T}$. Oosterloo, unpublished data

dwarf galaxy or of intergalactic clouds. In the case of a dwarf, the stellar component has either already fallen in and has been digested, or it has been totally disrupted and the stars are now scattered around. Deep optical imaging may be able to reveal them. Examples may be the unusual faint features around some galaxies reported by Malin and Hadley (1997) (see also below).

It is important to note that in all cases with no obvious interaction, a careful study of the structure and kinematics of the $\mathrm{HI}$ is necessary to distinguish between 

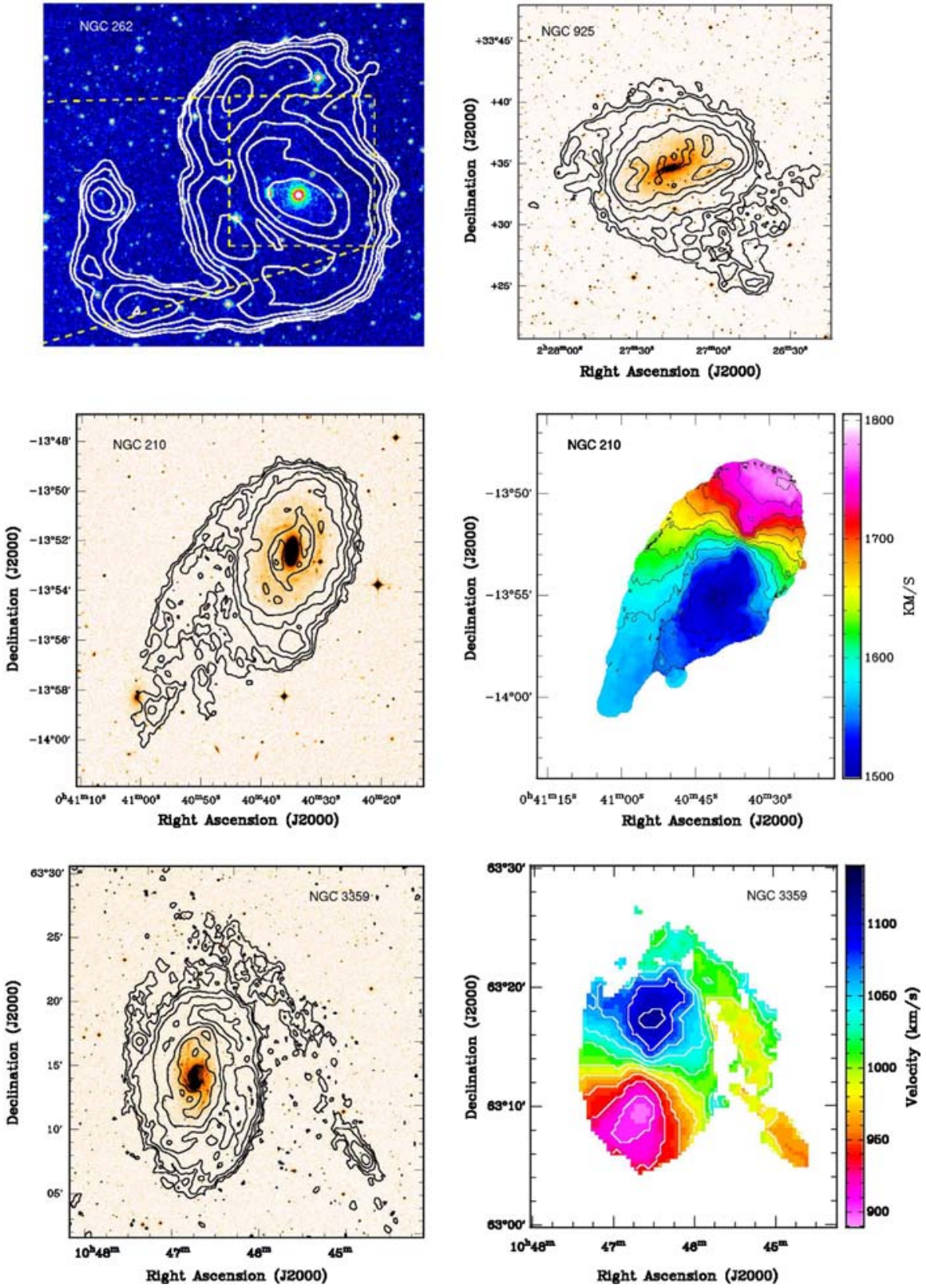

Fig. 1 Examples of galaxies showing signs of interactions/accretion. In each panel the contours show the HI density distribution superposed on the optical image. For NGC 925 the levels are 5, 10, 20, 50, 100 $\times 10^{19}$ atoms cm ${ }^{-2}$; for NGC 210: 5, 10, 20, 50, 100, $200 \times 10^{19}$ atoms cm ${ }^{-2}$; for NGC 3359: 10, 20, 50, $100,200,400 \times 10^{19}$ atoms $\mathrm{cm}^{-2}$. The HI data for NGC 262 are from Simkin et al. (1987). The middle and bottom right panels show the velocity fields of NGC 210 and NGC 3359 

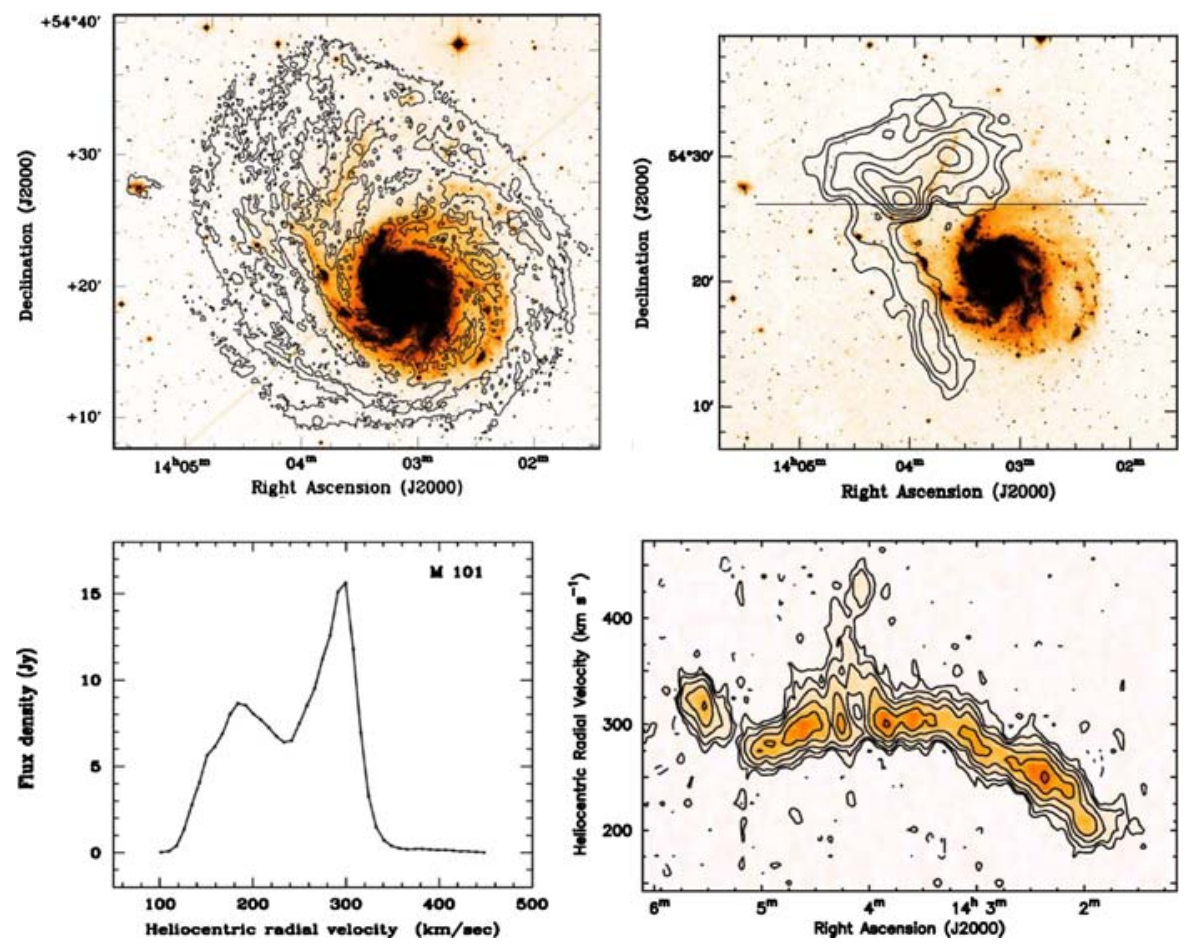

Fig. 2 Top left WSRT total H I map for M 101 (contours) overlaid on a DSS image. Top right high-velocity gas complex (contours) overlaid on the optical image. Bottom left global HI profile. Bottom right Positionvelocity diagram ( $45^{\prime \prime}$ beam) at constant declination (see horizontal line in top right panel) showing the high-velocity H I complex. The HI data are from Kamphuis (1993) (see also van der Hulst and Sancisi 1988)

configurations that can be considered "normal" and configurations that are definitely "peculiar" and point to a recent interaction and infall. There are recognizable signatures in the $\mathrm{HI}$ that make this distinction possible, but it is not always easy to draw the line between effects due to the internal metabolism of the galaxy and those due to the environment. As an example of this difficulty, it is interesting to consider lopsidedness, which affects spiral galaxies and seems to occur quite frequently (Sect. 5). Should it be attributed to past interactions and accretion events? This is not at all obvious and there may be other explanations related to the intrinsic dynamics of the system $(m=1$ mode) (Saha et al. 2007).

Recently, accretion of satellites has also been revealed by studies of the distribution and kinematics of stars in the halos of the Milky Way and of M31. The discovery of the Sgr Dwarf galaxy (Ibata et al. 1994) is regarded as proof that accretion is still taking place. Since such minor merger remnants retain information about their origin for a long time (Helmi and de Zeeuw 2000), studies of the distribution and kinematics of "stellar streams" can in principle be used to trace the merger history of the Milky Way (Helmi and White 2001). Stellar streams have also been discovered in M31 (Ibata et al. 2001; Ferguson et al. 2002; McConnachie et al. 2003). Such events 
are more difficult to trace in more distant galaxies, where it is not possible to observe individual stars. However, deep optical images of a number of spiral galaxies, such as NGC 253, M 83, M 104, NGC 2855, (Malin and Hadley 1997) and NGC 5907 (Shang et al. 1998), do show unusual, faint features in their surroundings. These galaxies do not have obvious interactions or companions (except NGC 5907 which has a nearby dwarf). To our knowledge, a clear association of these features with Hi has not been shown yet, except, perhaps, for the association between the Orphan stream and complex A discussed by Belokurov et al. (2007). It would be interesting to have deep optical images for H I systems like those illustrated in Fig. 1.

For the study of interactions and accretion, especially in the case of distant galaxies, the Hi has clear advantages. As has been shown for our galaxy and for nearby galaxies, Hi not only provides a direct measure of the accreting gas, but is also a powerful tracer of merger events. In particular, the Hi distributions and kinematics can be used for modeling and for estimating timescales. The improved sensitivity of modern synthesis radio telescopes brings within reach the detection of fainter and fainter $\mathrm{HI}$ signatures of accretion events and we expect that new observations of nearby and also more distant galaxies will reveal many more examples in the coming decade.

Neutral hydrogen found in early-type galaxies (E and S0) reveals a similar picture as illustrated above for spirals, indicating that the accretion phenomenon is probably playing an important role in all types of galaxies (see Table 1). Recent surveys of HI in early-type galaxies in the field (Oosterloo et al. 2007b; Sadler et al. 2001, 2002; Morganti et al. 2006) show that about $60-70 \%$ of them have detectable amounts of H I (detection limits $10^{7} \mathrm{M}_{\odot}$ ). Similar detection rates were found in E and S0 galaxies with optical fine structure, such as optical shells (Schiminovich et al. 1997; van Gorkom and Schiminovich 1997) (e.g. NGC 5128 (Cen A) (Schiminovich et al. 1994) and NGC 2865 (Schiminovich et al. 1995)), and also near ellipticals with dwarf companions, like NGC 4472 (McNamara et al. 1994) and NGC 3656 (Balcells and Sancisi 1996). About half of these galaxies do show apparently relaxed, gaseous disks in regular rotation. The other half have irregular $\mathrm{HI}$ distributions suggesting accretion and minor mergers in progress (Schiminovich et al. 1997; Oosterloo et al. 2007b). It is also interesting to note that there are gas-rich ellipticals, such as NGC 4278, that contain a lot of $\mathrm{HI}$ but have a purely old stellar population, indicating that gas accretion and star formation are not tightly correlated.

\subsection{Specific examples}

To further illustrate the processes of interaction with dwarf companions and of merger/ accretion we describe a few examples in detail.

\subsubsection{101}

In M 101 an $\mathrm{HI}$ complex of about $2 \times 10^{8} \mathrm{M}_{\odot}$ (Fig. 2, top right panel) has been found moving with velocities of up to $150 \mathrm{~km} \mathrm{~s}^{-1}$ with respect to the local disk and in correspondence with a large trough in the HI layer (Fig. 2, bottom right). It has been suggested that this is the result of a collision with a dwarf companion (not visible) 
or with a gas cloud complex which has gone through the Hi layer of M101 and has created the observed trough (van der Hulst and Sancisi 1988; Kamphuis 1993). The high-velocity gas will eventually rain back down onto the M 101 disk. It is interesting to note that M 101 is a prototype lopsided galaxy (Baldwin et al. 1980) (Fig. 2, top left; see Sect. 5). The lopsidedness is also manifested by the global Hi profile (Fig. 2, bottom left).

\subsubsection{NGC210}

NGC 210 is a good example of a galaxy that shows no indications of accretion or interaction in the optical, but where the H I data give clear evidence for a recent merging event. Figure 1 (middle left panel) shows that NGC 210 is a regular barred galaxy with well developed, symmetrical spiral arms. In the region of the optical disk, the $\mathrm{HI}$ contours indicate a regular structure. In the outer parts, however, the H I shows a long tail extending to the SE and containing about $6 \times 10^{8} \mathrm{M}_{\odot}$. This is $10 \%$ of the total HI mass of NGC 210. The kinematics of the tail is similar to that of the gas disk, i.e. the tail is co-rotating with the disk, at similar projected velocities (Fig. 1, middle right panel). Its orbital period is $1-2 \times 10^{9}$ years. This is about the time it will take for the asymmetric structure to wind up and disappear. Near the end of the tail, a small galaxy is visible in the optical. However, its redshift is known (da Costa et al. 1998) and shows that it is a background object at about ten times the distance of NGC 210, excluding an interaction. It is possible that the Hi tail is caused by a merger, although no signs of a victim are seen in optical data.

\subsubsection{NGC925}

Another example of a galaxy with an HI tail suggesting an accretion event is NGC 925. Figure 1 (top right) shows the $\mathrm{HI}$ distribution in relation to the optical image (see also Pisano et al. 1998). The H I properties are similar to those of NGC 210, and it appears that also NGC 925 has suffered an accretion event fairly recently. NGC 925 has a tail of $\mathrm{HI}$ extending to the South. The tail contains about $3 \times 10^{8} \mathrm{M}_{\odot}$ of $\mathrm{HI}$, or $5 \%$ of the total Hi mass of NGC 925. Also in NGC 925 the kinematics of the gas in the tail is not too different from that of the gas in the galaxy, i.e. it appears to co-rotate at similar projected velocities. The winding up and disappearance of this structure may take about $0.5-1.0 \times 10^{9}$ years. NGC 925 is different from, e.g. NGC 210 in that in the optical (Pisano et al. 2000) the galaxy is fraught with asymmetries, both morphological and kinematical, suggesting that the accreted object may have been relatively more massive than in NGC 210.

\subsubsection{NGC 3359}

NGC 3359 is a nearby SBc galaxy with a dynamical mass of $1.6 \times 10^{11} \mathrm{M}_{\odot}$ (Broeils and Rhee 1997) and an H I mass of $1.9 \times 10^{10} \mathrm{M}_{\odot}$. It has well developed spiral structure both in the optical and in HI. The observations (Kamphuis and Sancisi 1993; van der Hulst and Sancisi 2005) have revealed the presence of an H I companion and a long tail/bridge connecting to the outer spiral structure of NGC 3359 (bottom Fig. 1). 


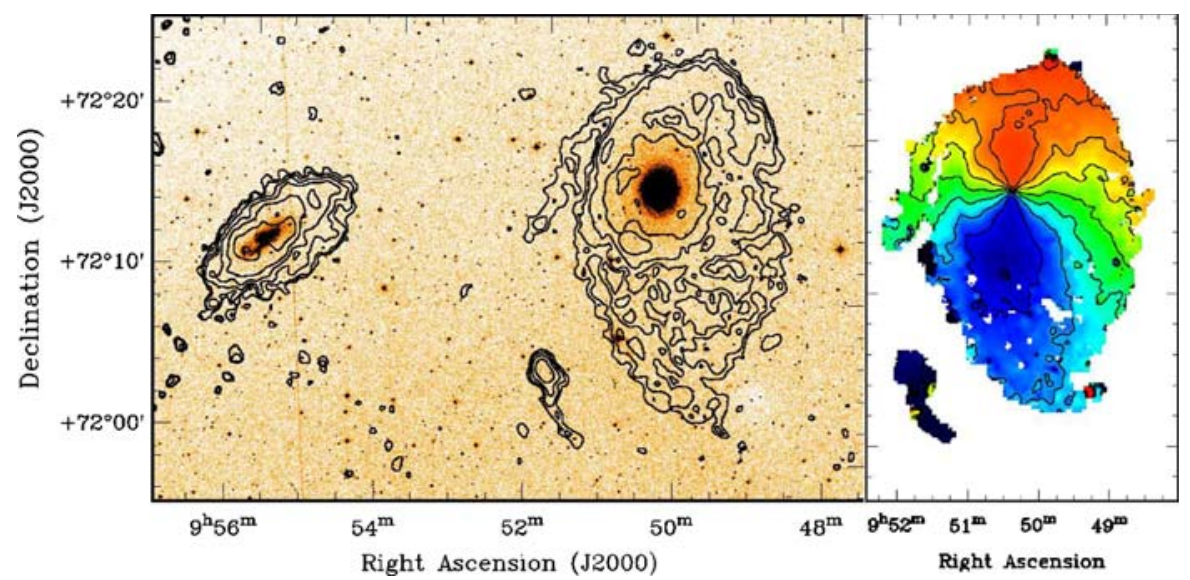

Fig. 3 Total H I map of NGC 2985 (UGC 5253) and its companions and velocity field. On the left plot, the shade is the DSS and contours are HI. Contour levels are 5, 10, 20, 50, 100, $200 \times 10^{19}$ atoms cm$^{-2}$

The companion has an $\mathrm{HI}$ mass of $4.4 \times 10^{8} \mathrm{M}_{\odot}$. There is also evidence for an optical counterpart. Together with the connecting structure the total HI mass is about $9.6 \times 10^{8} \mathrm{M}_{\odot}$ or $5 \%$ of the total HI mass of NGC 3359. The HI image of the companion is distorted. The velocity structure of the HI companion and the connecting H I (bottom right Fig. 1) fits in very well with the regular velocity field of NGC 3359. The regularity of the velocities suggests that the process has been going on slowly for at least about one rotational period (about $1.5 \times 10^{9}$ years).

\subsubsection{NGC2985 (UGC5253)}

Figure 3 shows the total HI map (contours) for NGC 2985 (the galaxy on the right) and its surroundings overlaid on a DSS image. NGC 2985 is a spiral galaxy at a distance of $18 \mathrm{Mpc}$. A number of features can be noted that are relevant here. First, the HI distribution is very asymmetric. This may be due to an interaction with NGC 3027, the galaxy 20' (120 kpc) east of NGC 2985 and of similar redshift as NGC 2985 (velocity difference $\sim 250 \mathrm{~km} \mathrm{~s}^{-1}$ ). NGC 3027 also has an asymmetric HI distribution. Interestingly, the velocity field of NGC 2985 is, overall, fairly regular and dominated by differential rotation (Fig. 3, right panel). It looks as if the passage of NGC3027 has caused a tidal displacement of the outer disk of NGC 2985 with respect to the inner disk without destroying it and making NGC 2985 very lopsided in appearance. The timescales for the disappearance of the asymmetry are about $1.5-3 \times 10^{9}$ years.

Another interesting feature is the small galaxy SE of NGC 2985. In the optical, there is a faint, low surface brightness object, coincident with the peak of the HI. The H I map shows a comet-like structure suggesting that this small galaxy is interacting with NGC 2985, losing part of the HI in the process. It is likely that this is an accretion of a small galaxy that we observe at an early stage of the process. NGC 2985 is very $\mathrm{HI}$ rich, its $\mathrm{HI}$ mass is $1.1 \times 10^{10} \mathrm{M}_{\odot}$. The HI mass of the small companion is 
about $3 \%$ of that of NGC 2985 , or $3.6 \times 10^{8} \mathrm{M}_{\odot}$, its systemic velocity is $185 \mathrm{~km} \mathrm{~s}^{-1}$ blue-shifted with respect to that of NGC 2985.

\subsubsection{NGC 4565}

NGC 4565 is a large edge-on galaxy of Hubble type Sb with a dwarf companion 6' $(\sim 30 \mathrm{kpc})$ to the north of the center, F378-0021557, which has $7.4 \times 10^{7} \mathrm{M}_{\odot}$ of H I compared to an HI mass of $2.0 \times 10^{10} \mathrm{M}_{\odot}$ for NGC 4565 (using a distance of $17 \mathrm{Mpc}$ ) (van der Hulst and Sancisi 2005). An Hi detection of this same companion has also been reported (with the name NGC 4565A) by Rupen (1991). Another companion, NGC 4562, somewhat more massive in $\mathrm{HI}\left(2.5 \times 10^{8} \mathrm{M}_{\odot}\right)$ and brighter optically is located $15^{\prime}(\sim 75 \mathrm{kpc})$ to the south-west of the center of NGC 4565 . The H I distribution, derived (by us) from a new sensitive observation with the Westerbork Synthesis Radio Telescope (WSRT) by Dahlem et al. (2005), is shown in Fig. 4 (top left panel) superposed on the DSS. The asymmetric warp is clearly visible.

Individual channel maps show low surface brightness HI emission to the north of the centre, in the direction of the faint companion F378-0021557. The HI emission from NGC 4565 in the velocity range from 1,250 to $1,290 \mathrm{~km} \mathrm{~s}^{-1}$ (close to the velocity of F378-0021557 and to the systemic velocity, 1,230 $\mathrm{km} \mathrm{s}^{-1}$, of NGC 4565) clearly shows distortions above the plane pointing towards the companion. This is seen in the map (Fig. 4, top right) showing the H I velocity structure and in the bottom panels of Fig. 4 which show two channel maps chosen at velocities in this range. In these maps one can clearly see the Hi layer bending towards F378-0021557, suggesting a connection with the dwarf and a strong disturbance in the H I disk of NGC 4565. While there seems to be little doubt that this bending of the HI layer is due to the interaction with the companion, it is not clear whether there is any relationship with the warp. It is likely that, eventually, the companion will merge with NGC 4565.

\subsubsection{NGC6946}

Figure 5 (top left) shows a total H I image of NGC 6946 down to column density levels of $1.3 \times 10^{19} \mathrm{~cm}^{-2}$ (Boomsma 2007). At about 36' ( 70 kpc) to the north-west there are two small companions. An intruiging feature in the Hi image of NGC 6946 is the plume to the north-west. This is $20 \mathrm{kpc}$ long and blends smoothly (also kinematically) with the HI disk of NGC 6946. A lower limit to its Hi mass is $7.5 \times 10^{7} \mathrm{M}_{\odot}$. This is similar to the HI masses of the companions $\left(1.2 \times 10^{8} \mathrm{M}_{\odot}\right.$ and $\left.8.8 \times 10^{7} \mathrm{M}_{\odot}\right)$. Although the plume is in the same range of radial velocities as the two companion galaxies there is no detected connection with them. As in many other cases of peculiar features found around galaxies, it is not clear what its origin is and whether this is new material arriving from outside or whether it is the result of a tidal perturbation of the outer gaseous layer of NGC 6946. It might even be the accretion, seen at an advanced stage, of a third companion galaxy similar in mass to the other two.

In NGC 6946 there is further evidence pointing to a possible infall of material from outside. In the first place there are the striking spiral arms in the outer Hi disk (see Sect. 3.1.5), well outside the bright stellar disk and the regions of star formation. Second, in the arm-interarm regions and following the spiral arms, there are strong 

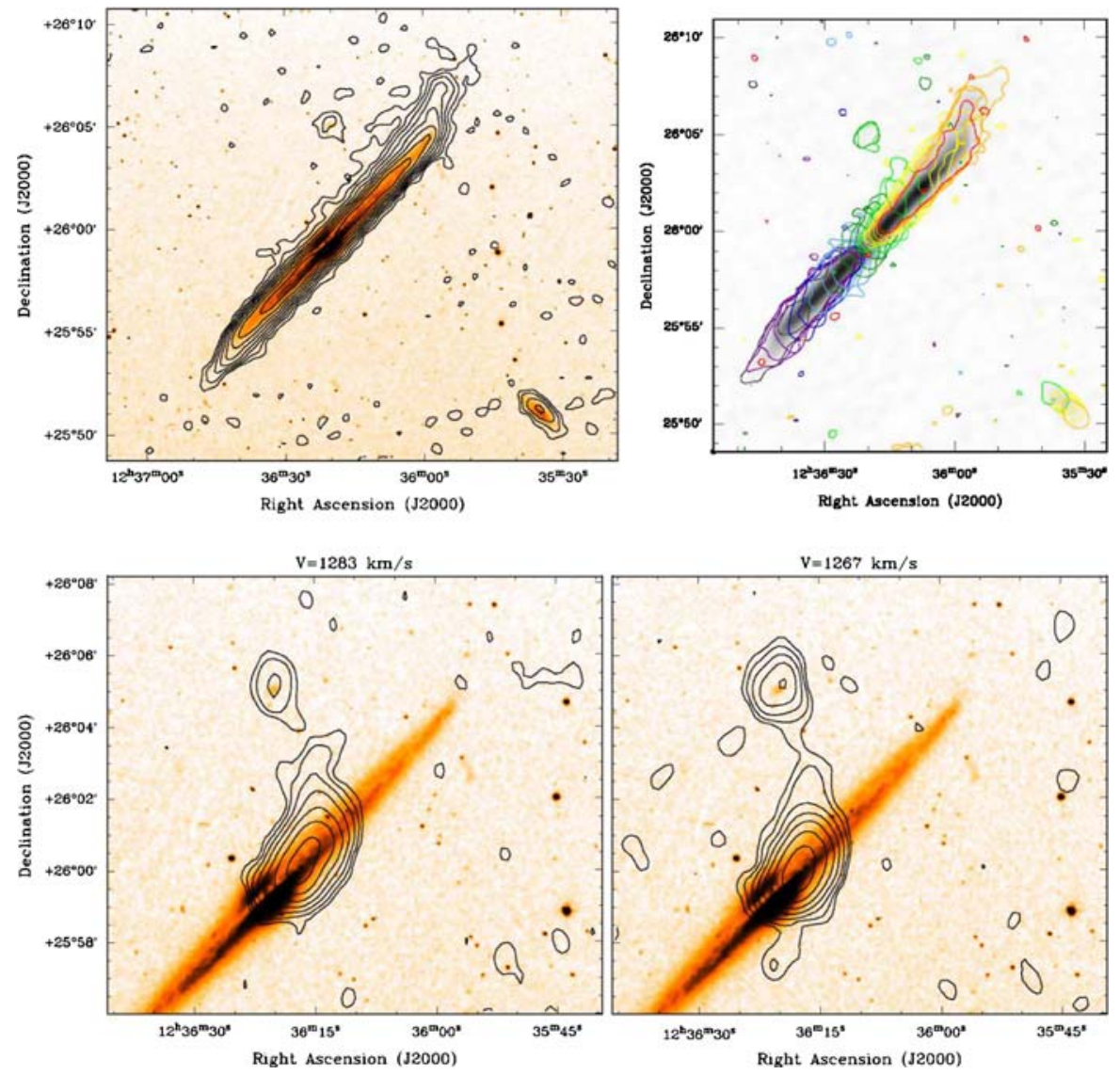

Fig. 4 Top left $\mathrm{HI}$ map of NGC 4565 at a resolution of $30^{\prime \prime}$ superposed on the DSS image. Contours are 2, $4,8,16,32$ and $64 \times 10^{20} \mathrm{~cm}^{-2}$. Top right outer contours of the H I emission in individual channels (from blue to $r e d$ ) superposed on the total HI density map. Bottom panels H I channel maps at two representative velocities superposed on the DSS image of NGC 4565. They clearly show the interaction between NGC 4565 and its small companion. Contours are 1, 2, 4, 8, 16, 32 and $64 \mathrm{mJy} / \mathrm{beam}$

velocity wiggles. These wiggles, clearly visible in the velocity field (Fig. 5, bottom left), are abrupt velocity deviations from circular motion reaching amplitudes of about $50 \mathrm{~km} \mathrm{~s}^{-1}$ (see $p-v$ plots in the right panels of Fig. 5). The corresponding troughs in the $\mathrm{HI}$ density distribution seem to indicate that the HI layer has been punched by infalling clumps of material. The picture is similar to that seen in M 101 and attributed to collision with intervening gas clouds.

The high resolution H I image of NGC 6946 is shown in Fig. 10, where it is compared with optical images, and the extra-planar $\mathrm{HI}$ is discussed below in Sect. 3.1.5.

\subsection{Frequency of accretion events}

How frequent are the interactions of galaxies with small companions and what is the fraction of galaxies with peculiar morphology and kinematics? What is the rate 

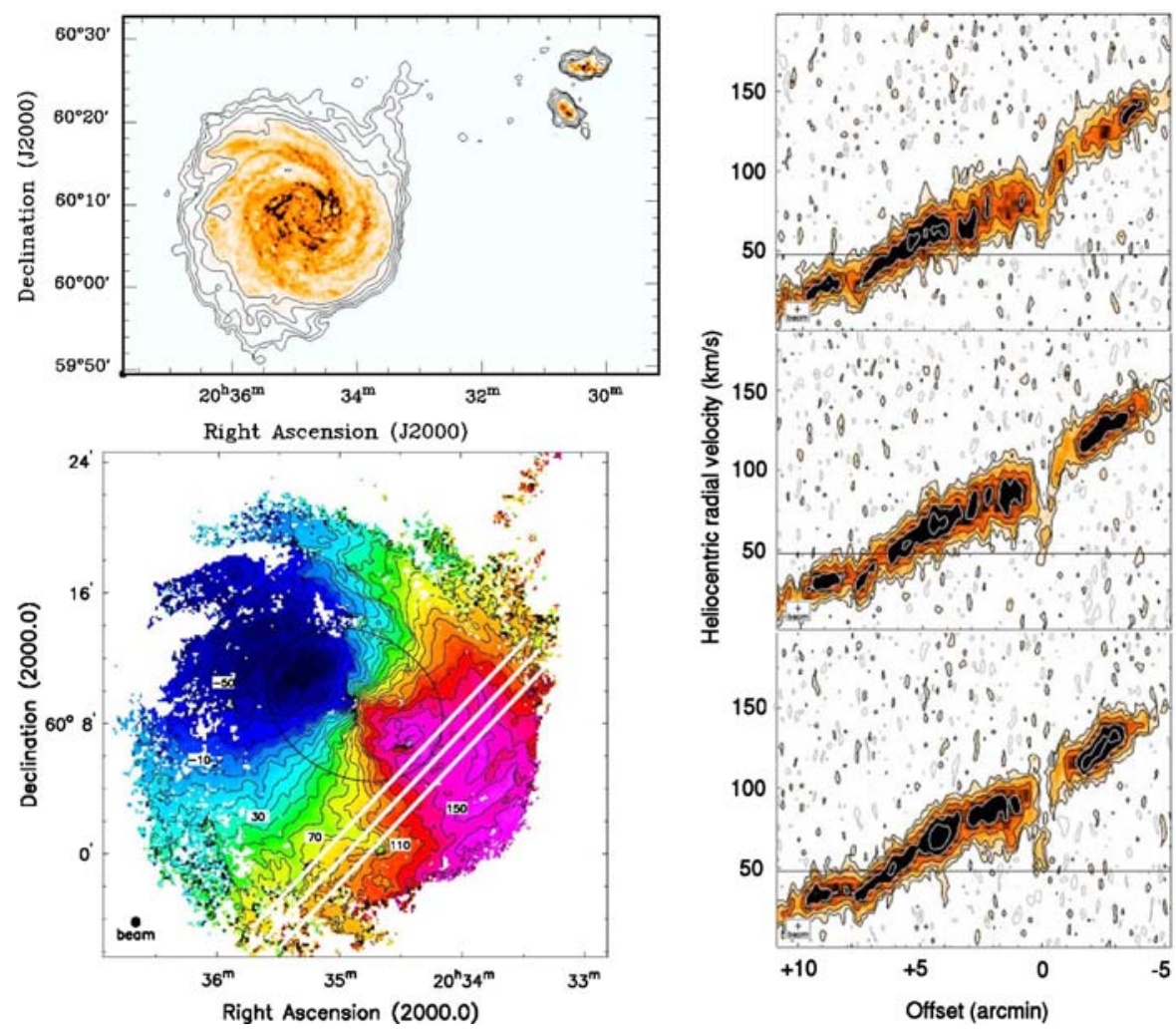

Fig. 5 Top left total H I map of NGC 6946 and two companions. The map has been corrected for primary beam attenuation. The shading shows the high resolution $\left(13^{\prime \prime}\right)$ HI map, the contours $(1.25,2.5,5,10$, and $20 \times 10^{19}$ atoms $\mathrm{cm}^{-2}$ ) show the low resolution $\left(60^{\prime \prime}\right) \mathrm{HI}$ distribution in the outer parts. Bottom left velocity field at $22^{\prime \prime}$ resolution The iso-velocity contours are separated by $10 \mathrm{~km} \mathrm{~s}^{-1}$ running from -70 (blue) to $150 \mathrm{~km} \mathrm{~s}^{-1}$. The small ellipse indicates the size and orientation of the optical disk $\left(R_{25}\right)$. Right panels position-velocity plots along the three white lines overlaid in the velocity field in the bottom left panel (Boomsma 2007)

of accretion expected from all these presumed minor mergers? In the past years, a large number of galaxies have been mapped in HI with the WSRT, the Very Large Array (VLA), the Australia Telescope Compact Array (ATCA) and the Arecibo radio telescope. A first estimate made on the basis of about one hundred galaxies led to the conclusion (Sancisi 1992) that at least $25 \%$ of field galaxies show signs of either present or recent tidal interactions. The incompleteness and inhomogeneity of the sample examined made such an estimate rather uncertain. An H I survey carried out for a magnitude and volume limited sample of galaxies from the Ursa Major cluster (Verheijen and Sancisi 2001) provides more solid statistical evidence on the frequency of tidal interactions and of accretion phenomena. This cluster differs from Virgo or Coma type clusters. It has a low velocity dispersion and long crossing time, comparable to the Hubble time. It has no central concentration and no detected X-ray emission and the sample is dominated by late-type systems. It can be considered, therefore, 
representative for a galaxy population in the field. Out of the 40 galaxies mapped in $\mathrm{HI}$, about ten show clear signs of interactions with small companions or have peculiar structures. About half of the sample galaxies show asymmetries in their kinematics or in the Hi density distribution. A larger sample of galaxies is the one provided by WHISP (van der Hulst et al. 2001). About $25 \%$ of 300 spirals and irregulars show evidence of minor interactions.

In conclusion, the available evidence from $\mathrm{HI}$ observations indicates that at least $25 \%$ of field galaxies are undergoing now or have undergone in the recent past some kind of tidal interaction. The lifetimes of the observed features are typically $\sim 1 \mathrm{Gyr}$. If lumps of gas with H I masses of order $10^{8-9} \mathrm{M}_{\odot}$ (as indicated by the $21 \mathrm{~cm}$ observations) are accreted at a rate of 1 per $10^{9}$ year, the mean accretion rate for the gas would be around $0.1-0.2 \mathrm{M}_{\odot}$ year $^{-1}$. This is certainly a lower limit for gas accretion as a fraction of the $\mathrm{HI}$ involved in the interaction may be undetected and neither ionized hydrogen nor helium have been considered. Furthermore, the number of past interactions and mergers may be higher. Indeed, if one is willing to accept also the lopsided structure and kinematics as evidence (see below), as also proposed in optical studies (Zaritsky and Rix 1997), then the conclusion would be that more than 50\% of present day galaxies have been through one or more merger events in a recent past. In such a case the accretion rate would be difficult to estimate, but it could be considerably higher than the values given above.

\section{Extra-planar $\mathrm{HI}$}

\subsection{Galaxies with Hi halos}

The presence of cold gas in the halo region of disk galaxies (extra-planar gas) is well established. For a small number of systems (Table 2) seen at various inclination angles

Table 2 Extra-planar gas in spiral galaxies

\begin{tabular}{llcllll}
\hline Galaxy & Type & Incl $\left(^{\circ}\right)$ & $\begin{array}{l}v_{\text {flat }} \\
\left(\mathrm{km} \mathrm{s}^{-1}\right)\end{array}$ & $\begin{array}{l}M_{\mathrm{HI}_{\text {halo }}} \\
\left(10^{8} \mathrm{M}_{\odot}\right)\end{array}$ & $\begin{array}{l}\frac{M_{\mathrm{HI}_{\mathrm{halo}}}}{M_{\mathrm{HI}}} \\
(\%)\end{array}$ & References \\
\hline Milky Way & $\mathrm{Sb}$ & - & 220 & $>0.2$ & $>1^{\mathrm{a}}$ & Wakker et al. (2007) \\
M31 & $\mathrm{Sb}$ & 77 & 226 & $>0.3$ & $>1$ & Thilker et al. (2004) \\
NGC 891 & $\mathrm{Sb}$ & 90 & 230 & 12 & 30 & Oosterloo et al. (2007a) \\
NGC 6946 & $\mathrm{Scd}$ & 38 & 175 & $>2.9$ & $>4$ & Boomsma et al. (2005b) \\
NGC 4559 & $\mathrm{Scd}$ & 67 & 120 & 5.9 & 11 & Barbieri et al. (2005) \\
NGC 2403 & $\mathrm{Scd}$ & 63 & 130 & 3 & 10 & Fraternali et al. (2002) \\
UGC 7321 & $\mathrm{Sd}$ & 88 & 110 & $\gtrsim 0.1$ & $\gtrsim 1$ & Matthews and Wood (2003) \\
NGC 2613 & $\mathrm{Sb}$ & $\sim 80$ & $\sim 300$ & $4.4^{\mathrm{b}}$ & 5 & Chaves and Irwin (2001) \\
NGC 253 & $\mathrm{Sc}$ & $\sim 75$ & $\sim 185$ & 0.8 & 3 & Boomsma et al. (2005a) \\
\hline
\end{tabular}

a Only HVCs (IVCs not included)

$\mathrm{b}$ From sum of the various extra-planar clouds 


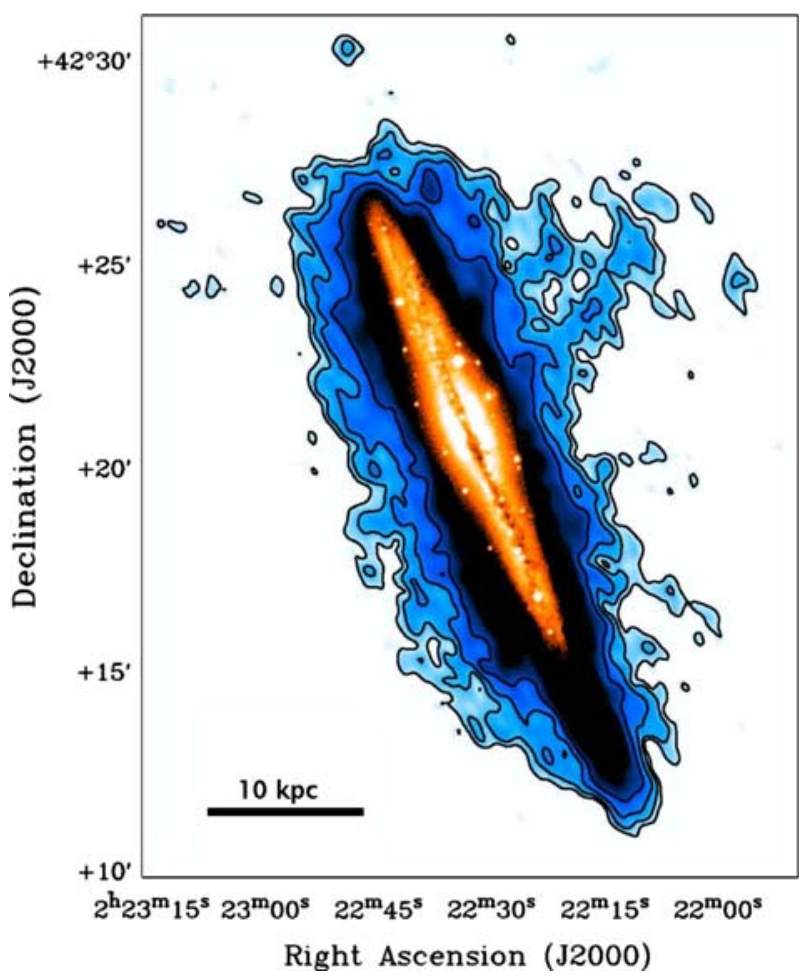

Fig. 6 Optical DSS image (red) and total H I map (contours + blue shade) of the edge-on galaxy NGC 891. HI contours are at $1,2,4,8,16 \times 10^{19}$ atoms $\mathrm{cm}^{-2}$ (Oosterloo et al. 2007a). The beam size is $25^{\prime \prime}=1.1$ $\mathrm{kpc}$

and, in particular, for the edge-on galaxy NGC 891 (see Fig. 6) there is now detailed information on the Hi structure and kinematics. This has been obtained from very deep observations with the WSRT and the VLA. Also the HVCs of our galaxy (at least the largest) are now regarded, as a result of the recent distance determinations (Wakker et al. 2007, 2008), as a Galactic halo population. The analogy between the Galactic HVCs and the high-velocity H I in external galaxies has been discussed by Oosterloo (2004).

What is the origin of the extra-planar gas? Undoubtedly, a large fraction has originated from the disk as an effect of star formation. The mechanism is that of a "galactic fountain" in which hot gas rises into the halo, condenses into cold clouds and returns to the disk (first suggested by Shapiro and Field 1976; see also Bregman 1980). There are various indications from the $\mathrm{H}$ I observations that extra-planar gas is indeed driven by star formation. One is the distribution of the extra-planar HI in NGC 891, which is concentrated very close to the star-forming disk (see Sect. 3.1.3). Another is the remarkable concentration of the majority of the high-velocity clouds in NGC 6946 (see Sect. 3.1.5) in the direction of the bright inner disk.

However, there is also evidence that a fraction of the extra-planar gas must be infall from intergalactic space. This is indicated primarily by the low metallicity of 
the HVCs, which points directly to an external origin: infall of pristine gas clouds or gas-rich dwarf companions. Similarly, in other galaxies, such infall is supported by the presence of huge Hi filaments and clouds with peculiar motions. An argument in favor of accretion may also come from the large-scale kinematics of the halo gas, which is characterized by rotational velocity gradients along the $z$ direction and a global inflow motion. It has been suggested that such kinematics can be explained by the interaction between the fountain gas and infalling gas, which carries low angular momentum (Fraternali and Binney 2008). However, the gradients by themselves may not necessarily require infall (e.g. Barnabè et al. 2006).

Here, we give a short review of the main, relevant observational results and we end with estimates of accretion rates. We describe the observations for the best cases known. For the edge-on galaxies (e.g. NGC 891) the extent and structure of the halo are observed directly and the rotational velocities are measured at various distances from the plane. In galaxies at lower inclination angles, such as NGC 2403 and NGC 6946, the presence of extra-planar gas is inferred from the observed anomalous kinematics (high velocities, slow rotation). Indeed, it is this anomalous kinematics that is used to separate the extra-planar gas from the thin disk. In particular, in NGC 6946, which is closer to face-on, one sees a large number of clouds with high velocities, i.e. with large velocity deviations from circular motion, which can be unambiguosly separated from the differentially rotating disk. It should be emphasized that all these galaxies are not undergoing strong gravitational interactions and, therefore, the observed high-velocity structures are not tidal features.

\subsubsection{Milky Way}

The HVCs of neutral hydrogen in our galaxy (Wakker and van Woerden 1997) (see also recent review by van Woerden et al. 2004) (Fig. 7, top panel) have been considered since their discovery as possible direct evidence for infalling gas. The lack of information on their distances and therefore on their masses has been, however, a major obstacle. It has even been proposed that they are a population of clouds in the Local Group of Galaxies (Blitz et al. 1999). Recently, it has been possible, through the study of absorption lines in the spectra of stars, to set distance brackets on some of the largest and more massive clouds like complex A (8-10 kpc, van Woerden and Wakker 2004) and complex C (3.7-11.2 kpc, Wakker et al. 2007). For this latter, the conclusion is that it must be located high above the Galactic plane $(\mathrm{z}=3-9 \mathrm{kpc})$ and at a Galactocentric radius $\mathrm{R}<14 \mathrm{kpc}$. Its mass estimate is $3-14 \times 10^{6} \mathrm{M}_{\odot}$, its metallicity is 0.15 times solar. Such low metallicities, also found for other HVCs (van Woerden and Wakker 2004), establish that most of this gas must be fairly pristine and hence of external origin (from gas-rich dwarfs or intergalactic clouds) and not ejected from the disk. The mass inflow is estimated at $0.1-0.25 \mathrm{M}_{\odot}$ year $^{-1}$, including ionized hydrogen and a $40 \%$ contribution from helium. The masses and sizes of these HVC complexes are similar to those of the filaments found in NGC 891 and in NGC 2403 and reported below. The IVCs form a component closer to the Galactic layer. It is now clear that IVCs and HVCs are a halo population of the Milky Way, analogous to the gaseous halos found in external galaxies. Indeed, seen from outside, 

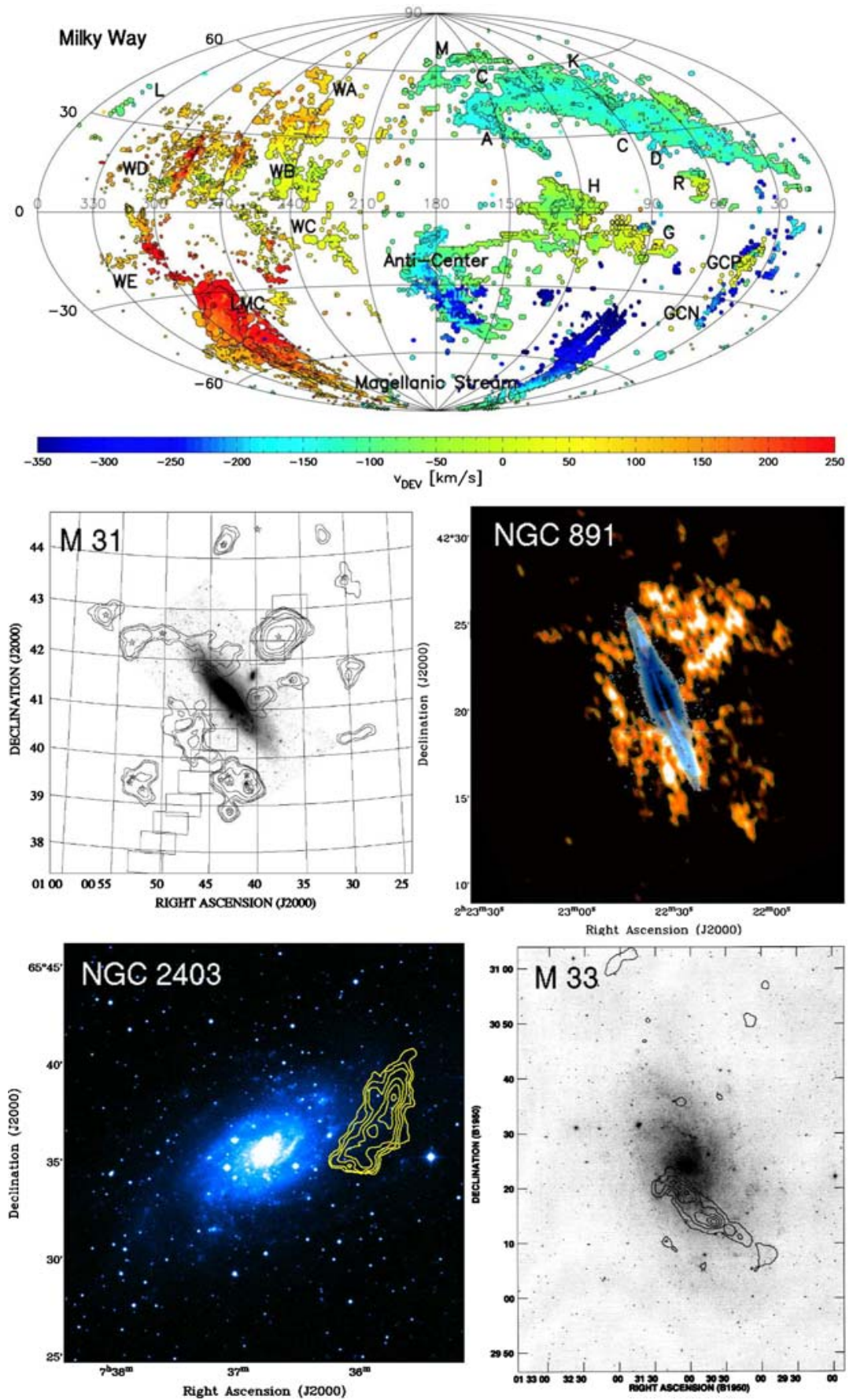

Fig. 7 Examples of extra-planar gas complexes around nearby galaxies. From top High-Velocity Clouds in the Milky Way, extra-planar gas features in M31 and NGC 891. The bottom panels show two massive HI filaments in NGC2403 and M33 
the halo of our galaxy (IVCs included) might well look like the halos of NGC 891 and NGC 2403.

\section{$3.1 .2 \mathrm{M} 31$}

H I observations with the Green Bank Telescope (GBT) by Thilker et al. (2004) have revealed a population of faint $\mathrm{HI}$ clouds (at least 20) surrounding M31 within 50 $\mathrm{kpc}$ of its disk (Fig. 7, middle left) and with radial velocities comparable to those of the outer disk rotation. The masses of these clouds are in the range $10^{5}-10^{7} \mathrm{M}_{\odot}$. In addition, a filamentary component of at least $30 \mathrm{kpc}$ extent is concentrated at the M31 systemic velocity. Thilker et al. argue that all this gas is associated with M31. The total amount of $\mathrm{HI}$ for the halo cloud population within the GBT field is estimated to be $\sim 3-4 \times 10^{7} \mathrm{M}_{\odot}$, which is only $1 \%$ of the mass of the HI disk of M31. This is probably a lower limit. For the origin of this M31 halo Thilker et al. suggest various possibilities: a Local Group cooling flow, tidal debris from recent mergers or the gaseous counterpart of low-mass dark matter halos.

\subsubsection{NGC 891}

The nearby edge-on galaxy NGC 891 has been observed in H I a number of times over the past three decades with increasing sensitivity (Sancisi and Allen 1979; Rupen 1991; Swaters et al. 1997; Oosterloo et al. 2007a). With the sensitivity improvement by about a factor of 50 from the first to the latest observations (Fig. 6, see also Oosterloo et al. 2007a) it has been possible to trace the Hi emission in the vertical direction to $22 \mathrm{kpc}$ from the plane, whereas the size of the Hi disk (as measured along the major axis) has remained unchanged (Oosterloo et al. 2007a). Radially, the halo extends to the end of the disk on the N-E side but stops earlier on the S-W side where the disk is more extended. This is an indication that the main bulk of the halo is closely connected to the inner disk of NGC 891 where star formation is higher. The mass of the Hi halo is about $1.2 \times 10^{9} \mathrm{M}_{\odot}, \sim 30 \%$ of the total HI mass. Its kinematics is characterized by a slower rotation with respect to the disk, with a vertical gradient of $-15 \mathrm{~km} \mathrm{~s}^{-1} \mathrm{kpc}^{-1}$ (Fraternali et al. 2005). The same gradient has been found also in the ionized gas (Heald et al. 2006). In addition there is, at velocities close to systemic, a remarkable filament extending up to $22 \mathrm{kpc}$ from the plane and containing $1.6 \times 10^{7} \mathrm{M}_{\odot}$. There are also other structures and in particular some clouds with anomalous (counter-rotating) velocities and masses of $1-3 \times 10^{6} \mathrm{M}_{\odot}$.

In Fig. 7, we show the optical picture of NGC 891 surrounded by the Hi left after subtraction of a "normal" (symmetrical, smooth, regular) disk and halo component (models from Oosterloo et al. 2007a, Fig. 14). The features seen here, some of them (e.g. the filament) recognizable in Fig. 6, represent the H I around NGC 891 which is the most peculiar for its location and kinematics. Their total mass is about $1 \times 10^{8} \mathrm{M}_{\odot}$. We believe that these features may be regarded as the analogue of the HVCs in the Milky Way.

NGC 891 has also a small, gas-rich companion (UGC 1807) at about $80 \mathrm{kpc}$ (projected distance) and $100 \mathrm{~km} \mathrm{~s}^{-1}$ higher radial velocity and with about one tenth of the total mass of NGC 891. 

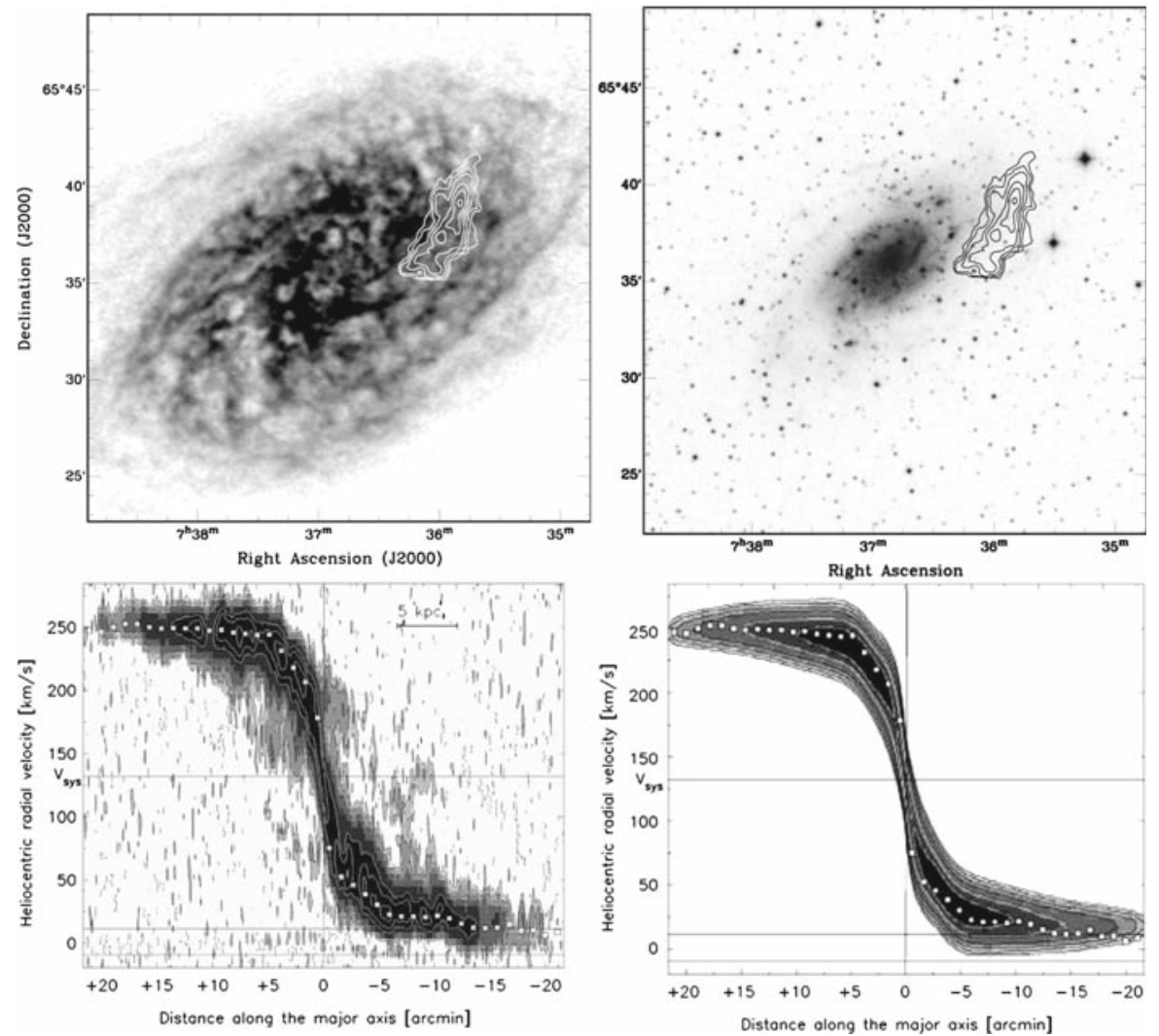

Fig. 8 Top panels total HI (VLA) map (left) and optical DSS image (right), on the same scale, for the spiral galaxy NGC2403. The contours show a 8-kpc long HI filament kinematically decoupled from the bulk of the disk gas. Bottom panels comparison between the H I position-velocity plot along the major axis of NGC 2403 and the prediction for a thin disk model (right panel). The gas at anomalous velocities, not visible in the model, is extra-planar gas (Fraternali et al. 2002)

\subsubsection{NGC 2403}

For other galaxies seen at intermediate inclinations [NGC 2403 (Schaap et al. 2000; Fraternali et al. 2002) and NGC 4559 (Barbieri et al. 2005)] or more "face-on" (M 101 and NGC 6946) the presence of gaseous halos has been inferred from the anomalous velocities. In NGC 2403 and NGC 4559 the signature is the asymmetric velocity shape of the HI line profiles (Fig. 8, bottom left). The deep HI observations of NGC 2403 with the VLA and a careful 3-D modeling (Fraternali et al. 2001) have led to conclusions very similar to those obtained for the edge-on NGC 891. Also in NGC 2403 there is a vertically extended (a few $\mathrm{kpc}$ ) gas component with lagging rotation. It has a total mass of about $3 \times 10^{8} \mathrm{M}_{\odot}(\sim 10 \%$ of the total HI mass $)$ and contains long filamentary structures. In addition to its lagging rotation, the halo gas has a large-scale inflow motion of about $15 \mathrm{~km} \mathrm{~s}^{-1}$ toward the center of the galaxy. 
The most striking filament (Fig. 8 upper right, and Fig. 7 bottom left) has coherent (narrow) velocity structure (with radial velocities close to systemic), it is $8 \mathrm{kpc}$ long and contains about $1 \times 10^{7} \mathrm{M}_{\odot}$. It is seen projected on the outer parts of NGC 2403, beyond the bright optical disk. This is similar to the filament found in the halo of NGC 891 and to the one found in M33 (Fig. 7, bottom right) (J.M. van der Hulst, unpublished data). It is also close in size and mass to the largest Milky Way's HVCs like complex C. Such filaments are the most remarkable and extreme structures found in the halo regions of these galaxies (Fig. 7). They may be quite common among spirals and be direct evidence of cold gas accretion from intergalactic space (see Sect. 3.2).

\subsubsection{NGC 6946}

NGC 6946 is a bright, nearby spiral galaxy of Hubble type Scd (inclination angle $38^{\circ}$ ) which has been studied in H I several times (Rogstad et al. 1973; Tacconi and Young 1986; Boulanger and Viallefond 1992; Kamphuis 1993). It was in this galaxy that Kamphuis and Sancisi (1993) found evidence for an anomalous-velocity H I component which they interpreted as an outflow of gas from the disk into the halo as a result of stellar winds and supernova explosions. A more recent, very sensitive and detailed study of the anomalous H I and of the H I disk has been carried out by Boomsma (2007) with the WSRT. The density distribution in the H I disk is characterized by the presence of a large number of holes of diameters up to $2 \mathrm{kpc}$. The average amount of H I missing from each hole is about $1 \times 10^{7} \mathrm{M}_{\odot}$, the total amount is $1.1 \times 10^{9} \mathrm{M}_{\odot}$. Widespread high-velocity gas has been detected amounting to a total of $2.9 \times 10^{8} \mathrm{M}_{\odot}(4 \%$ of the total HI mass) and deviating by $>50 \mathrm{~km} \mathrm{~s}^{-1}$ from local disk rotation. This gas is made of clumps and filaments of various sizes and most of it is seen projected against the $\mathrm{H} \alpha$ bright inner disk of NGC 6946, which suggests a close relationship with the regions of star formation. A large part of it must be in the halo of NGC 6946, although its distance from the plane is not known. It follows the galaxy differential rotation but there are clear indications that it is rotating more slowly than the gas in the disk. Overall, the picture is very similar to that of NGC 2403, and the conclusion reached by Boomsma (2007) is that the disk of NGC 6946 is surrounded by a lagging H i halo similar to the halos found for NGC 891 and NGC 2403. The outer parts of the disk of NGC 6946 are discussed in Sect. 2.2.7.

\subsubsection{Other galaxies}

There is, in addition to the galaxies described above, a number of other objects in which extra-planar gas (or traces of it) has been found. Some of these are low surface brightness (LSB) galaxies, others have high surface brightness as those just described above or are even starbursts.

Matthews and Wood (2003) find evidence for vertically extended H I emission up to $2.4 \mathrm{kpc}$ in the edge-on, superthin LSB galaxy UGC 7321. They find tentative evidence that the vertically extended gas has declining rotational velocity as a function of $z$. They estimate the Hi mass of the halo (above $\sim 1.4 \mathrm{kpc}$ ) to be $\sim 1 \times 10^{7} \mathrm{M}_{\odot}$. This value is approximately 15 times lower than the fractional $\mathrm{HI}$ content (ratio between gas above $1.4 \mathrm{kpc}$ and gas in the disk) at comparable $z$-heights in NGC 891. In a 
similar, superthin LSB galaxy, IC 2233, Matthews and Uson (2008) report a component of "anomalous" extra-planar gas. Also in the LSB galaxy NGC 4395 (see Sect. 5), Heald and Oosterloo (2008) find H I cloud complexes with anomalous velocities, and presumably located in the halo, with masses of a few $\times 10^{6} \mathrm{M}_{\odot}$ each and, in total, about $5 \%$ of the $\mathrm{HI}$ content of that galaxy.

In the starburst galaxy NGC 253, Boomsma et al. (2005a) find extra-planar HI concentrations reaching as high as $12 \mathrm{kpc}$ above the disk. The gas seems to be lagging in rotation and has a total Hi mass of $8 \times 10^{7} \mathrm{M}_{\odot}$. In the spiral galaxy NGC 4559, Barbieri et al. (2005) find extra-planar HI with properties similar to those found for NGC 2403. Several high-latitude Hi features are observed in NGC 5775 (Lee et al. 2001) and NGC 2613 (Chaves and Irwin 2001; Irwin and Chaves 2003); however, in the first case there is evidence for a strong interaction with the companion galaxy NGC 5774 (Irwin 1994).

\subsection{Accretion rate}

The fraction of Hi present in the halo of spiral galaxies seems to vary considerably from galaxy to galaxy (see Table 2). However, only for a few cases there are good estimates of the amounts of $\mathrm{HI}$ gas in the halo. These range from about $3 \times 10^{8} \mathrm{M}_{\odot}$ for NGC $2403\left(\sim 10 \%\right.$ of the total HI) (Fraternali et al. 2002) to $1.2 \times 10^{9} \mathrm{M}_{\odot}$ (about $30 \%$ of total) found for NGC 891 (Oosterloo et al. 2007a). In our galaxy, the HVCs probably are only a small fraction of the extra-planar $\mathrm{HI}$ and should be regarded as an "extreme" population. Clearly, for a better estimate and a proper comparison with external galaxies such as NGC 891, one should include the IVCs together with the HVCs.

To date, the number of objects studied with sufficient sensitivity and angular resolution is very limited and it is not possible yet to investigate possible dependencies on morphology, luminosity, surface brightness or star formation activity. However, concerning the origin of extra-planar gas, there seems to be little doubt that, in high surface brightness galaxies, galactic fountains are responsible for a large part of it; but accretion from IGM is also taking place. Low surface brightness galaxies are more intriguing: galactic fountains, if present at all, are expected to play a less important role and yet the observations of the LSB galaxies UGC 7321, IC 2233 and NGC 4395 do reveal the presence of some extra-planar gas. Unfortunately, most of these observations are not deep enough to trace the halo emission to large heights and to obtain a good estimate of the extra-planar Hi mass.

Accretion rates for extra-planar gas have been estimated using different techniques. Values for the gas accretion rate around $0.1-0.25 \mathrm{M}_{\odot}$ year $^{-1}$ have been reported for the HVCs of our galaxy (Wakker et al. 2007). These include ionized gas and helium. The observations of some of the galaxies discussed here (NGC 891, NGC 2403 and M33) have revealed the presence of filamentary structures in their halos which are, like the HVCs, most likely of external origin. The main argument in favor of this interpretation is their high kinetic energy requirement (of order $10^{4}-10^{5}$ supernovae). Moreover, some gas complexes are observed at forbidden ("counter-rotating") velocities (see e.g. Oosterloo et al. 2007a), which suggests material not dynamically linked 
to the galaxy disk. One expects that the metallicities (not known) of all these filaments and gas complexes are as low as those found for the HVCs. If we assume that all these features are accreted gas, we get values for the "visible" accretion rate of 0.1$0.2 \mathrm{M}_{\odot}$ year $^{-1}$ (H I only), similar to the values obtained for the HVCs of the Milky Way. This is of the order of 10\% the SFR in spirals like NGC 891 and NGC 2403, and also in the Milky Way.

The actual accretion rate may, however, be significantly higher and reach values approximately equal to the SFR, as a large fraction of the accreting gas may already be mixed with the fountain gas coming from the disk. Such mixing could solve the problem of the peculiar kinematics of the extra-planar gas (negative rotational gradients and inflow) which cannot be reproduced in purely galactic fountain models (Collins et al. 2002; Heald et al. 2006; Fraternali and Binney 2006). Fraternali and Binney (2008) have explored this possibility by including accretion of low angular momentum gas from the IGM in their models, and have calculated the amount of accreting material necessary to reproduce the observations of NGC 891 and NGC 2403. They have found that the accretion rates needed are very close to the respective SFRs.

In conclusion, the extra-planar gas seems to consist of two parts: a large one from galactic fountains and a smaller part accreted from intergalactic space. There is direct (HVCs in our galaxy and filaments in external galaxies) and indirect (rotational velocity gradients) evidence for the accretion from outside. Accretion rates range from a minimum of about $0.1-0.2 \mathrm{M}_{\odot}$ year $^{-1}$ to values possibly ten times higher, as needed to meet SFR requirements. It is clear that the observed extra-planar gas cannot be purely and totally made up from accretion: on the one hand, accretion rates would be unrealistically high $\left(\sim 30 \mathrm{M}_{\odot}\right.$ year $^{-1}$ for NGC 891$)$, and, on the other, there is strong observational evidence that galactic fountains do take place and have a major part in building up the halo (see Sect. 3.1).

\section{Extended, warped H I outer disks}

The H I disks of the large majority of spiral galaxies are known to extend considerably beyond the bright optical disk. Broeils and Rhee (1997) find for the ratio of the HI radius (defined at a surface density level of $1 M_{\odot} \mathrm{pc}^{-2}, N_{\mathrm{H}}=1.3 \times 10^{20}$ atoms cm $^{-2}$ ) to the optical radius $\left(R_{25}\right)$ an average value $R_{\mathrm{HI}} / R_{25}=1.7 \pm 0.5$. In some cases the H I extends much further out, to several optical radii. Striking examples are DDO 154 (Krumm and Burstein 1984), NGC 4449 (Bajaja et al. 1994), NGC 2915 (Meurer et al. 1996), and NGC 3741 (Begum et al. 2005; Gentile et al. 2007). This large extent of the $\mathrm{H}$ I disks has made it possible to trace the rotation curves far beyond the bright stellar disk and thus provide the crucial evidence for the existence of dark matter in spiral galaxies. We consider the possibility that these outer layers have accumulated from tidal debris or infall of gas clouds, as described in Sect. 2, and that they now form a reservoir of fresh gas for fuelling star formation in the inner regions.

Here, we show the HI images of the blue compact dwarf galaxy NGC 2915 (Fig. 9, Meurer et al. 1996) and of the two spiral galaxies NGC 6946 (Fig. 10, Boomsma 2007) and NGC 5055 (Fig. 11, Battaglia et al. 2005). Generally, in the galaxies studied with sufficient resolution, the outer Hi layers show spiral features (see e.g. NGC 5055). 


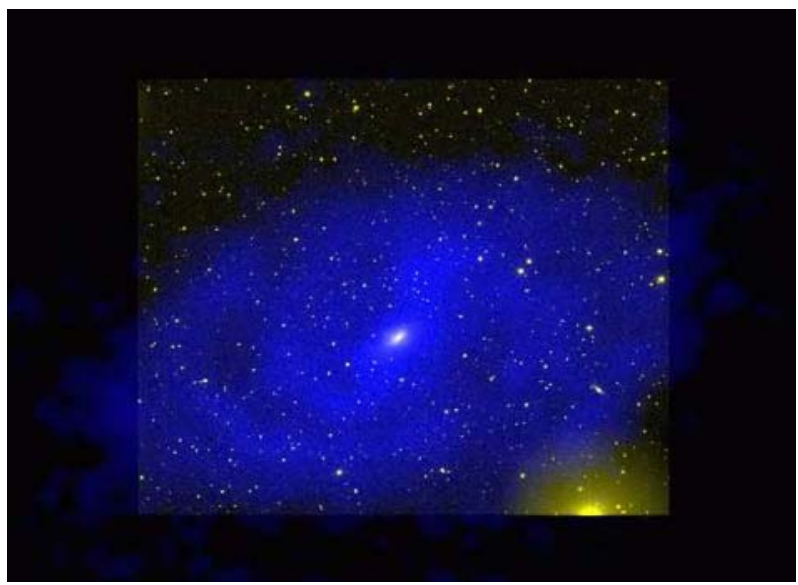

Fig. 9 Composite optical (yellow) and H I (blue) image of the dwarf galaxy NGC 2915 from Meurer et al. (1996)
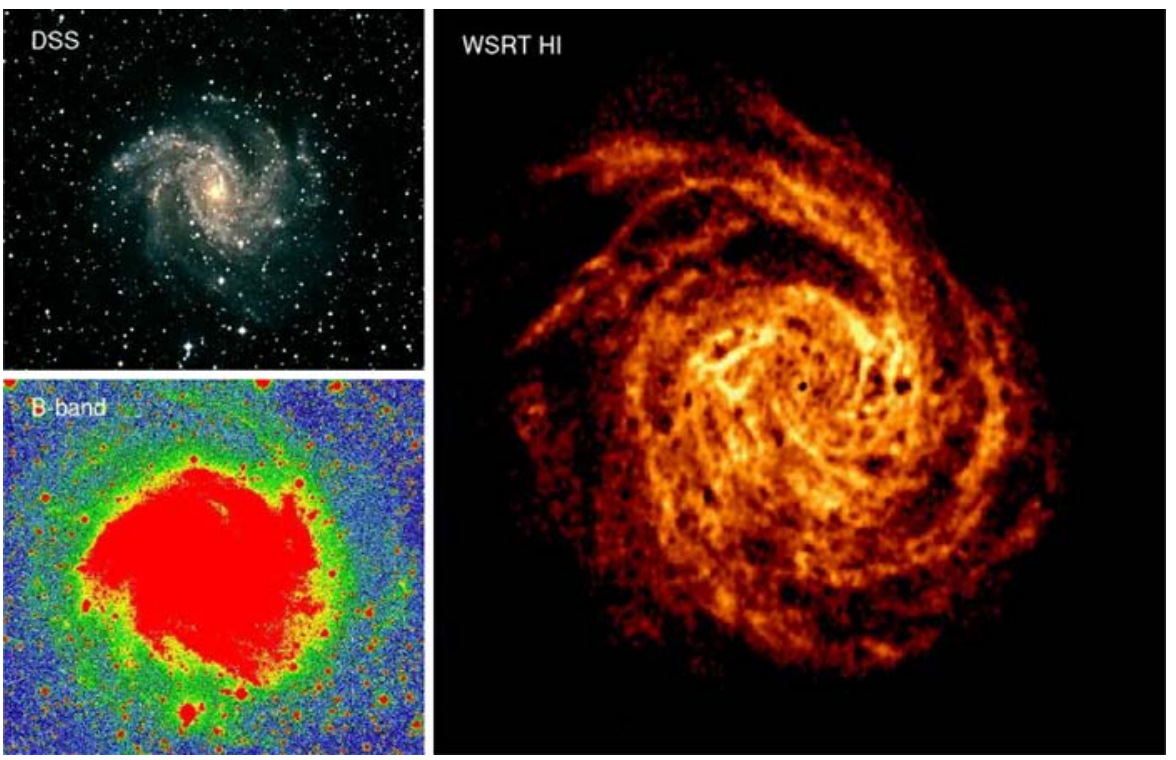

Fig. 10 Comparison between optical images and total H I map for NGC6946. All images are on the same scale. Top left color composite of the Digitized Sky Survey plates. Bottom left deep B-band image from Ferguson et al. (1998). Right deep WSRT total HI map from Boomsma (2007). Column densities range from $6 \times 10^{19}$ atoms $\mathrm{cm}^{-2}$ to $3 \times 10^{21}$ atoms $\mathrm{cm}^{-2}$

Strong spiral arms are seen in the outer parts of NGC6946; well-developed arms are also observed in the low luminosity galaxies NGC 2915 and NGC 3741 (Gentile et al. 2007). It is remarkable that there is such pronounced spiral structure in the outer regions of spirals where dark matter dominates and even in the dwarfs where the dark halo is believed to be predominant everywhere. The question is, therefore, whether 


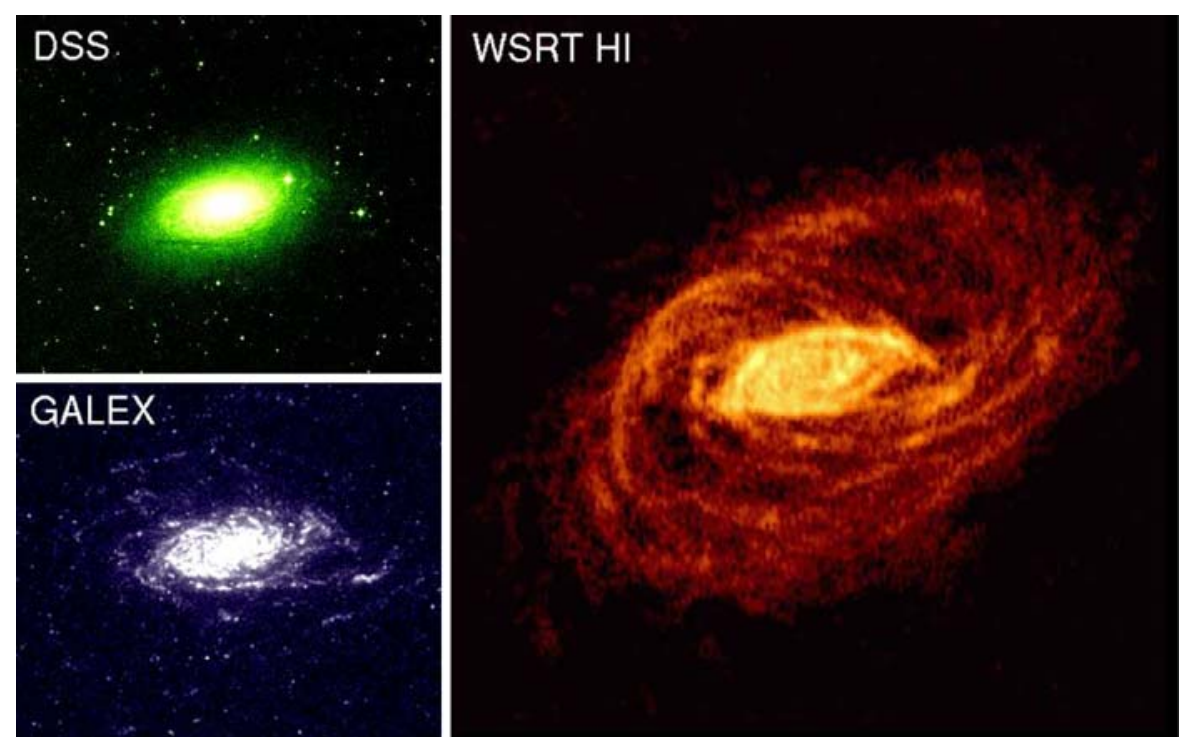

Fig. 11 Optical (DSS), GALEX and WSRT HI images of the warped galaxy NGC 5055 (all on the same scale). Column densities range from about $3 \times 10^{19}$ atoms cm $\mathrm{cm}^{-2}$ to $1 \times 10^{21}$ atoms cm ${ }^{-2}$ (Battaglia et al. 2005)

these systems have light disks surrounded by massive dark halos or, rather, have heavy and dark disks.

These gaseous outer parts of disks are usually warped. This is especially clear in edge-on galaxies. In systems viewed at lower inclination angles, the warping is inferred from the velocity field. Prominent examples of warps are those of the edge-on galaxies NGC 5907 (Sancisi 1976; Shang et al. 1998) and NGC 4013 (Bottema 1996), shown in Fig. 12, and of the less inclined galaxy NGC 5055 (Battaglia et al. 2005) shown in Fig. 11. These are also among the most symmetrical. In general, warps are quite asymmetric, like the warp of our galaxy. Warps seem to be quite common: according to Bosma (1991) the fraction of warped Hi disks is at least 50\%. A recent Hi study of 26 edge-on galaxies (García-Ruiz et al. 2002) has shown that 20 galaxies are warped and that all galaxies with H I extending beyond the stellar disk are warped to some degree. There are also edge-on galaxies, such as NGC 4565, which clearly show the presence of an optical warp. As a result of a statistical study of 540 edge-on galaxies, Reshetnikov and Combes (1999) present a sample of 60 of the clearest and strongest among the $174 \mathrm{~S}$-shape optical warps found. The existence of stellar warps is not surprising. In a galaxy seen less inclined, such as NGC 5055, it is possible to see from its GALEX image (Fig. 11, see also Thilker et al. (2007)) that stars have formed in the warped gaseous layer, in correspondence with the Hi spiral features. The systematic properties of warps have been investigated by Briggs (1990) and more recently by Jozsa (2007).

Although various possibilities for the origin and persistence of warps have been discussed, there still is no satisfactory explanation. The possibility that warps are the consequence of accretion of gas with a slewed angular momentum due to cosmic infall has been suggested by Ostriker and Binney (1989) and by Jiang and Binney (1999). 

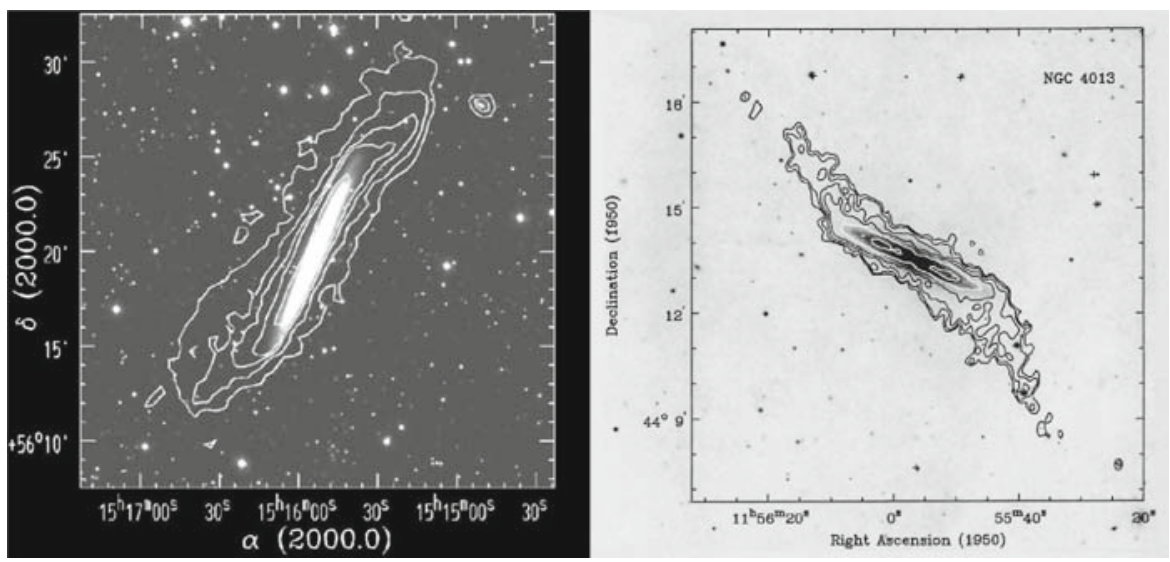

Fig. 12 Total HI maps (contours) of two warped edge-on galaxies overlaid on optical images. Left NGC 5907 from Shang et al. (1998). Right NGC 4013 from Bottema (1996)

The amount of Hi located in the outer parts of spiral galaxies, beyond $R_{25}$, is on average approximately equal to the amount of $\mathrm{HI}$ in the bright inner stellar disk (Broeils and Rhee 1997). It ranges, therefore, from $10^{8}$ to $10^{10} \mathrm{M}_{\odot}$ from the small to the large galaxies. Potentially, this gas represents a huge reservoir available for the replenishment of the inner disk where the star-formation rate is higher. As far as we know, however, there is no direct evidence for a radial inflow of this cold gas toward the centre. In the case of NGC 2403 such a radial inflow, of about $15 \mathrm{~km} \mathrm{~s}^{-1}$ (Fraternali et al. 2002), has been found for the halo gas, but not for the disk. The H I velocity fields would reveal the presence of large-scale inflows larger than $5 \mathrm{~km} \mathrm{~s}^{-1}$. The signature is the non-orthogonality of major and minor axis. To our knowledge, no such inflow motions have been reported. In order to flow inward, the outer gas has to loose part of its angular momentum. It is not clear how this can happen and, therefore, whether such a process of inflow would work. An overall radial motion is probably unrealistic. However, often the outer H I is not in circular orbits: bars, oval distortions and lopsided structures (see Sect. 5) are very common and may play a role. As an example, to reach an infall rate of $1 \mathrm{M}_{\odot}$ year $^{-1}$ one would need to move about $2 \times 10^{9} \mathrm{M}_{\odot}$ of gas from the outer to the inner parts with a radial inward speed of $5 \mathrm{~km} \mathrm{~s}^{-1}$. This would take about $2 \times 10^{9}$ years.

In conclusion, the possibility envisaged here is that gas accreted from satellites or directly from the IGM is deposited in the outermost parts of galactic disks. These outer layers of gas and, to some extent, also stars are characterized by spiral features and large-scale warping. They form a reservoir of gas which in turn could, from there, slowly migrate to replenish the inner parts of galaxies.

\section{Lopsidedness}

Infall of substantial amounts of gas and stars may have observable effects on the disks of spiral galaxies. One of these is the lopsidedness in the H I density distribution and in 

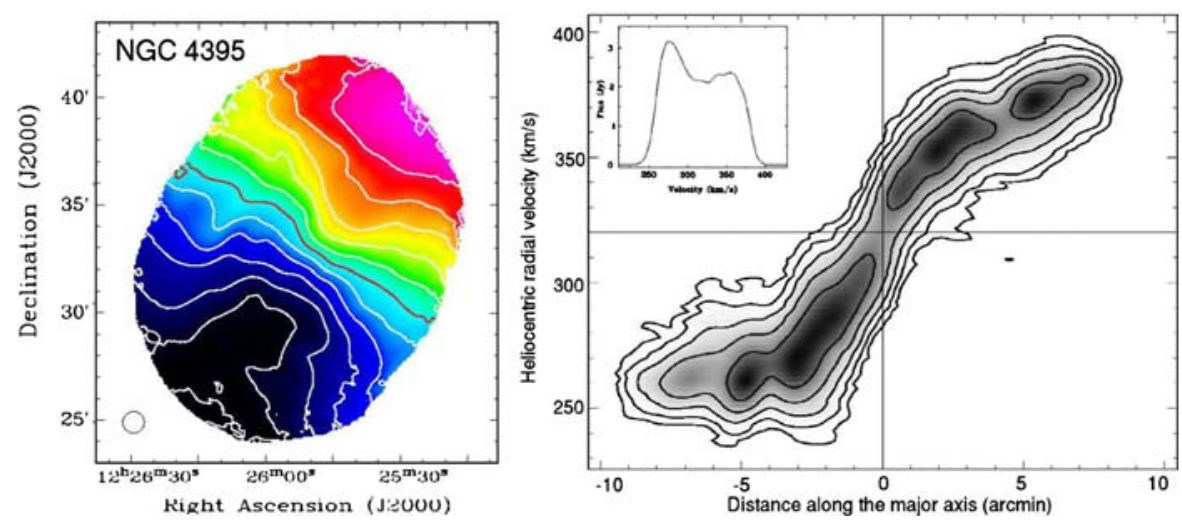

Fig. 13 Left velocity field of NGC4395 at $1^{\prime}$ resolution (blue is approaching). The contours are at $10 \mathrm{~km} \mathrm{~s}^{-1}$ intervals and range from $260 \mathrm{~km} \mathrm{~s}^{-1}$ (SE) to $380 \mathrm{~km} \mathrm{~s}^{-1}$ (NW). Right position-velocity plot along the major axis of NGC 4395 and global HI profile (Heald and Oosterloo 2008, in preparation)

the disk kinematics, which has been known for many years and seems to be a common phenomenon among spirals. Since the first study (Baldwin et al. 1980), based on a small number of objects, much new evidence has become available. The frequency of asymmetries among spiral galaxies has been estimated from the global Hi profiles of a large sample of field galaxies (Richter and Sancisi 1994). Examples of asymmetric global Hi profiles are shown in Fig. 2 for M 101 and in Fig. 13 for NGC 4395. About $20 \%$ of the systems examined showed strong asymmetries and up to more than $50 \%$ of the whole sample showed some mild asymmetries. This result has been confirmed by a $21-\mathrm{cm}$ H I survey of 104 isolated galaxies with the Green Bank 43-m telescope (Haynes et al. 1998) and also by more recent observations which image the HI distribution and the kinematics of a large sample of galaxies (WHISP). At least one half of about 300 objects from WHISP shows some lopsidedness either in the H I distribution or in the kinematics or in both. Deviations from axial symmetry seem, therefore, to be the rule rather than the exception. It should be noted that, in general, these lopsided galaxies are not interacting systems and that, therefore, the lopsidedness cannot be explained as a present tidal effect.

The lopsidedness is generally present in the HI density distribution as in M 101 (see Fig. 2) and also in the kinematics. In some cases, such as NGC 4395 (see Fig. 13), the asymmetry is only seen in the kinematics: on one side of the galaxy the rotation curve rises more slowly (almost solid body) and reaches the flat part at larger radii than on the other side. This is the signature of the kinematic lopsidedness as discussed by Swaters et al. (1999). In such cases there seems to be a well-defined pattern that pervades the whole velocity field and may be related to a lopsided potential.

Although the morphological lopsidedness is most clearly seen in the HI data, there is often evidence of asymmetries also in the distribution of light, in the B-band as well as in the I and K bands (Rix and Zaritsky 1995; Zaritsky and Rix 1997; Kornreich et al. 1998). One of the most striking cases is that of the spiral galaxy NGC 1637 (Fig. 14), which is exceptionally lopsided in blue light as well as in the near infrared (Block et al. 1994), indicating that the lopsidedness is present not only in the young stellar population but also in the old stellar disk. In this galaxy there is clear evidence 

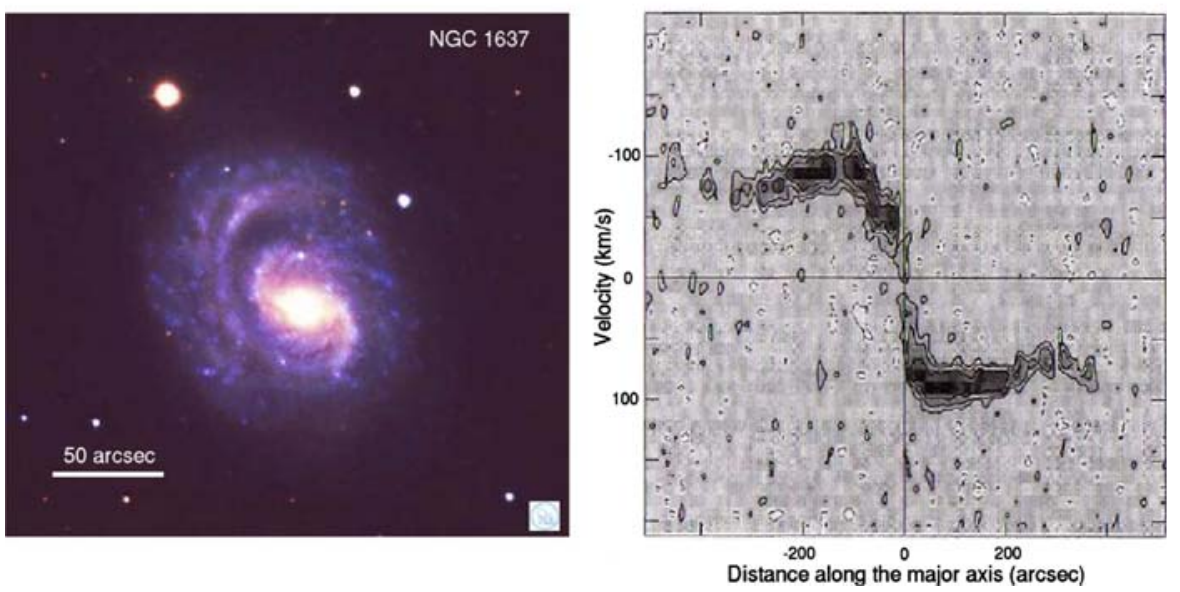

Fig. 14 Left optical image of NGC 1637. Right position-velocity plot along the major axis of NGC 1637 (Roberts et al. 2001), North is on the left

for a kinematical anomaly associated with the morphological asymmetry. Indeed, H I observations with the VLA (Roberts et al. 2001) reveal a peculiarity in the velocity structure in correspondence with the northern anomalous arm. This shows up as a step in the position-velocity diagram along the major axis (at about $-60^{\prime \prime}$ in the $p-v$ diagram in Fig. 14) implying a local deviation of at least $50 \mathrm{~km} \mathrm{~s}^{-1}$ from circular motion. This peculiar feature closely resembles the strong kinematic anomaly and the strong H I arm in the southern part of M 101 (Kamphuis 1993). This suggests that the lopsidedness of NGC 1637 is not only manifest in the optical appearance, but it is also present in the disk dynamics. As Block et al. (1994) point out, this is either an extreme $m=1$ asymmetry, and in such case its persistence should be explained, or it is the effect of a recent tidal interaction. We note that no companion for NGC 1637 is found on the Palomar Observatory Sky Survey within one degree.

All these facts suggest that the phenomenon of lopsidedness in spiral galaxies is quite common and structural for the disk. It is not clear what its origin is and how persistent it is. Minor mergers (Zaritsky and Rix 1997) and tidal interactions (Kornreich et al. 2002) have been suggested as possible causes. Bournaud et al. (2005), however, have found from numerical simulations that galaxy interactions and mergers are not sufficient to explain all the properties of the observed lopsided galaxies and have concluded that cosmological accretion of gas on galactic disks must be responsible. At any rate, it seems likely that many of the asymmetries, especially some of those revealed by the Hi distribution and kinematics, as for instance in M 101, are transient phenomena and are due to recent accretion events such as those described above in Sect. 2.

\section{Intergalactic H I clouds}

In the previous sections, we have discussed direct and indirect evidence on the accretion of cold gas in galaxies. We have argued that part of this accretion may come from 
the merging with gas-rich dwarf companions and part may come directly from the IGM in the form of gas clouds or filaments without stellar counterpart. It is natural, therefore, to ask what is the role of dwarfs in bringing in the gas and whether H I clouds exist in intergalactic space, away from galaxies.

Several observations of the fields around galaxies and of groups similar to the Local Group have been undertaken (Zwaan et al. 2001; de Blok et al. 2002; Pisano et al. 2004, 2007) as well as large blind surveys, such as HIPASS (Barnes et al. 2001) and the ongoing large survey ALFALFA (e.g. Giovanelli et al. 2005). Most of these observations have a sensitivity to detect clouds of $\mathrm{HI}$ with a mass of about $10^{7} \mathrm{M}_{\odot}$ (assuming a profile width of $30 \mathrm{~km} \mathrm{~s}^{-1}$ ). One survey (Kovač 2007) has the sensitivity (over large volumes of space) to detect smaller objects, down to masses of $10^{6} \mathrm{M}_{\odot}$.

The results can be summarized as follows:

(i) There is no evidence for a significant population of isolated Hi clouds (so-called dark galaxies) in the IGM. Kovač (2007) finds no clouds without optical counterpart down to $10^{6} \mathrm{M}_{\odot}$. The first results from ALFALFA show that only 3\% of their H I sources are not detected in the optical (Haynes 2007; Giovanelli et al. 2007). There is a hint in Kovač's survey, that galaxies with low H I masses are missing in the Local Volume. This could indicate that there is a lower limit to the gas mass of field galaxies of around a few times $10^{6} \mathrm{M}_{\odot}$.

(ii) In galaxy groups no clouds are detected, with detection limits of $10^{7} \mathrm{M}_{\odot}$. By analogy, this is an argument against the hypothesis that the HVCs are members of the Local Group with masses above $10^{7} \mathrm{M}_{\odot}$ (Blitz et al. 1999), in line with the recent distance determinations (Wakker et al. 2007, 2008) and with the conclusion that HVCs are located in the Galactic halo (see Sect. 3.1.1) and have masses lower than $10^{7} \mathrm{M}_{\odot}$.

These results also agree with what is known from the very deep H I observations of some nearby galaxies, like those of NGC 891 and NGC 6946 reported above. Although the areas surveyed around these galaxies are not very extended, as they usually correspond to only a few times the galactic $\mathrm{HI}$ images, they do seem to be empty and, remarkably, all the clouds detected (with masses of $10^{6}-10^{7} \mathrm{M}_{\odot}$ ) are located very close to or within the halo regions of the galaxies. Yet, some of these clouds are likely to have an intergalactic origin and not to be the result of galactic fountains.

To sum up, there is no evidence for the presence of a population of gas clouds in intergalactic space capable of accounting for the gas now observed near galaxies (halos and surroundings) and, above all, capable of fuelling the whole process of star formation.

Could all the gas needed for star formation have been brought in by dwarf companions? The HI mass function shows that most of the HI in the local universe is in large galaxies, with masses above about $10^{9} \mathrm{M}_{\odot}$ (Zwaan et al. 2005). If we assume that large galaxies accrete gas only via minor mergers with smaller galaxies and we require that the rate of gas accretion is about $1 \mathrm{M}_{\odot}$ year $^{-1}(\sim \mathrm{SFR})$, then all small galaxies would have been accreted by the large galaxies in a timescale of the order of $1 \times 10^{9}$ year. Clearly, an accretion rate of $1 \mathrm{M}_{\odot}$ year $^{-1}$ purely in the form of small galaxies cannot be sustained for much longer than one Gyr. This is inconsistent with the constancy 
of star formation in the Milky Way and also with the results of absorption studies of Damped Lyman Alpha (DLA) systems (Prochaska et al. 2005), which show that the cosmic integral H I mass density and the column density distribution of galaxies have evolved very little in the last 10 Gyr (Zwaan et al. 2005).

It is clear from the above considerations that constant gas accretion rates as high as required for star formation cannot be reached via mergers with satellite galaxies and that, therefore, a substantial amount of gas accretion must come directly from the IGM. Most of this accretion must take the form of gas clouds (or filaments). The reason why such clouds are not observed in intergalactic space remains an open question. Possibilities are that: (i) the clouds are confined near the galaxies by the hot galactic halos, whereas they disperse in the IGM; (ii) the clouds are intrinsically very small (smaller that $\approx 10^{6} \mathrm{M}_{\odot}$ ) and the accretion takes the form of a continuous drizzle of gas. Note that only the Hi masses have to be small and a certain amount of dark matter cannot be excluded. (iii) H I clouds are produced by large-scale cooling flows of the IGM in the regions surrounding the galaxies (Kaufmann et al. 2006; Peek et al. 2007).

\section{Conclusion}

We have drawn attention to a number of results from $\mathrm{H}$ I observations of nearby spiral galaxies which bear directly or indirectly on cold gas accretion. There are large H I complexes, with and without stellar counterparts, in the neighborhood of galaxies and in close interaction with them. There is little doubt about their extragalactic origin. Moreover, many galaxies have extra-planar gas components. Although most of this extra-planar gas must come from galactic fountains, a fraction of it, being in filaments and massive clouds like the Galactic HVCs, is likely to have an external origin. From the study of the gas seen in the neighborhood of galaxies and in their halos (the fountain gas excepted) we estimate a "visible" accretion rate of at least $0.2 \mathrm{M}_{\odot}$ year $^{-1}$.

Furthermore, there are large amounts of $\mathrm{HI}$, in the mass range $10^{8-10} \mathrm{M}_{\odot}$, in the warped outer galactic disks which could be a gas reservoir for replenishing the inner parts and fuelling star formation. In some cases (e.g. NGC 5055) it is obvious that this gas, because of its symmetrical and regular structure and long timescales, must have been there for a long time (several Gyr). It is conceivable, however, that in other cases the outer gas layers have been accumulated from outside more recently and that warping and spiral arms are related to such a process. Finally, there is the phenomenon of disk lopsidedness, which is poorly understood, but may be pointing to recent infall. Although both the warped outer layers and the lopsidedness may well be the effect of accretion from intergalactic space, estimates of infall rates are difficult to obtain.

The visible gas accretion rate, estimated above, does not seem to be sufficient to account for the star formation in galaxies $\left(\approx 1 \mathrm{M}_{\odot}\right.$ year $\left.{ }^{-1}\right)$. Direct, indisputable evidence for the required levels of cold gas accretion does not exist. There is, however, the puzzle of the peculiar kinematics of the extra-planar gas (overall negative vertical gradients in the rotational velocities and inflow motion) that could be solved by invoking infall of gas from outside carrying low angular momentum. It seems that this would give accretion rates of the order of the SFRs. 
Finally, the question arises of where the infalling gas, we hypothesized above, could originate from. Gas-rich dwarfs seem only to be able to account for a small fraction of the required gas and thus most of the accretion should come directly from the IGM. There is, however, no evidence for a population of large H I clouds in intergalactic space, in regions away from galaxies. Therefore, how and in what form gas infall onto galaxies takes place remains an open question and a challenge for future investigations.

Acknowledgments We thank Rense Boomsma for providing the NGC 6946 images and George Heald for the NGC 4395 figure. We are grateful to James Binney, Raffaella Morganti, Eline Tolstoy, Monica Tosi and Martin Zwaan for helpful comments and stimulating discussions.

Open Access This article is distributed under the terms of the Creative Commons Attribution Noncommercial License which permits any noncommercial use, distribution, and reproduction in any medium, provided the original author(s) and source are credited.

\section{References}

Bajaja E, Huchtmeier WK, Klein U (1994) The extended HI halo in NGC 4449. A\&A 285:385

Balcells M, Sancisi R (1996) Gas accretion in NGC 3656 (ARP 155). AJ 111:1053

Baldwin JE, Lynden-Bell D, Sancisi R (1980) Lopsided galaxies. MNRAS 193:313

Barbieri CV, Fraternali F, Oosterloo T, Bertin G, Boomsma R, Sancisi R (2005) Extra-planar gas in the spiral galaxy NGC 4559. A\&A 439:947

Barnabè M, Ciotti L, Fraternali F, Sancisi R (2006) Hydrostatic models for the rotation of extra-planar gas in disk galaxies. A\&A 446:61

Barnes DG, Staveley-Smith L, de Blok WJG, Oosterloo T, Stewart IM, Wright AE, Banks GD, Bhathal R, Boyce PJ, Calabretta MR, Disney MJ, Drinkwater MJ, Ekers RD, Freeman KC, Gibson BK, Green AJ, Haynes RF, te Lintel Hekkert P, Henning PA, Jerjen H, Juraszek S, Kesteven MJ, Kilborn VA, Knezek PM, Koribalski B, Kraan-Korteweg RC, Malin DF, Marquarding M, Minchin RF, Mould JR, Price RM, Putman ME, Ryder SD, Sadler EM, Schrder A, Stootman F, Webster RL, Wilson WE, Ye T (2001) The Hi Parkes All Sky Survey: southern observations, calibration and robust imaging. MNRAS 322:486

Battaglia G, Fraternali F, Oosterloo T, Sancisi R (2005) HI study of the warped spiral galaxy NGC 5055: a disk/dark matter halo offset? A\&A 447:49

Begum A, Chengalur JN, Karachentsev ID (2005) A dwarf galaxy with a giant HI disk. A\&A 433:L1

Belokurov V, Evans NW, Irwin MJ, Lynden-Bell D, Yanny B, Vidrih S, Gilmore G, Seabroke G, Zucker DB, Wilkinson MI, Hewett PC, Bramich DM, Fellhauer M, Newberg HJ, Wyse RFG, Beers TC, Bell EF, Barentine JC, Brinkmann J, Cole N, Pan K, York DG (2007) An Orphan in the "Field of Streams". ApJ, 658:337

Binney JJ, Dehnen W, Bertelli G (2000) The age of the solar neighbourhood. MNRAS 318:658

Blitz L, Spergel DN, Teuben PJ, Hartmann D, Burton WB (1999) High-Velocity Clouds: building blocks of the local group. ApJ 514:818

Block DL, Bertin G, Stockton A, Grosbol P, Moorwood AFM, Peletier RF (1994) $2.1 \mathrm{~m}$ images of the evolved stellar disk and the morphological classification of spiral galaxies. A\&A 288:365

Bond JR, Cole S, Efstathiou G, Kaiser N (1991) Excursion set mass functions for hierarchical Gaussian fluctuations. ApJ 379:440

Boomsma R (2007) The disk-halo connection in NGC 6946 and NGC 253. Ph.D. thesis. University of Groningen

Boomsma R, Oosterloo TA, Fraternali F, van der Hulst JM, Sancisi R (2005a) Extra-planar HI in the starburst galaxy NGC 253. A\&A 431:65

Boomsma R, Oosterloo T, Fraternali F, van der Hulst JM, Sancisi R (2005b) High velocity HI in NGC 6946 and Extra-planar Gas in NGC 253. In: Braun R (ed), Extra-planar Gas, Dwingeloo, ASP conference Series, vol 431, p 65

Bosma A (1991) Warped and flaring HI disks. In: Casertano S, Sackett P, Briggs F (eds). Warped disks and inclined rings around galaxies. Cambridge University Press, Cambridge, p 181

Bottema R (1996) The prodigious warp of NGC 4013. III. Analysis of the structure and kinematics. A\&A 306:345 
Boulanger F, Viallefond F (1992) Observational study of the spiral galaxy NGC 6946. I-HI and radiocontinuum observations. A\&A 266:37

Bournaud F, Combes F, Jog CJ, Puerari I (2005) Lopsided spiral galaxies: evidence for gas accretion. A\&A 438:507

Braun R, Walterbos RAM, Kennicutt RC, Tacconi LJ (1994) Counterrotating gaseous disks in NGC 4826. ApJ 420:558

Bregman JN (1980) The galactic fountain of high-velocity clouds. ApJ 236:577

Briggs FH (1990) Rules of behavior for galactic WARPS. ApJ 352:15

Broeils AH, Rhee M-H (1997) Short 21-cm WSRT observations of spiral and irregular galaxies. HI properties. A\&A 324:877

Brüns C, Kerp J, Staveley-Smith L, Mebold U, Putman ME, Haynes RF, Kalberla PMW, Muller E, Filipovic MD (2005) The Parkes HI Survey of the Magellanic System. A\&A 432:45

Carilli CL, van Gorkom JH (1992) HI imaging of four quasar-galaxy pairs-The parent galaxies of lowredshift quasar absorption systems. ApJ 399:373

Chaves TA, Irwin JA (2001) High-latitude HI in NGC 2613: structure and dynamics. ApJ 557:646

Collins JA, Benjamin RA, Rand RJ (2002) Kinematics of diffuse ionized Gas halos: a ballistic model of halo rotation. ApJ 578:98

da Costa LN, Willmer CNA, Pellegrini PS, Chaves OL, Rité C, Maia MAG, Geller MJ, Latham DW, Kurtz MJ, Huchra JP, Ramella M, Fairall AP, Smith C, Lípari S (1998) The Southern Sky Redshift Survey. AJ 116:1

Dahlem M, Ehle M, Ryder SD, Vlajić M, Haynes RF (2005) Neutral hydrogen gas in 7 high-inclination spiral galaxies. I. The data. A\&A 432:475

de Blok WJG, Zwaan MA, Dijkstra M, Briggs FH, Freeman KC (2002) An HI survey of the Centaurus and Sculptor groups. Constraints on the space density of low mass galaxies. A\&A 338:43

Dekel A, Birnboim Y (2006) Galaxy bimodality due to cold flows and shock heating. MNRAS 368:2

Ferguson AMN, Wyse RFG, Gallagher JS, Hunter DA (1998) Discovery of recent star formation in the extreme outer regions of disk galaxies. ApJ 506:19L

Ferguson AMN, Irwin MJ, Ibata RA, Lewis GF, Tanvir NR (2002) Evidence for Stellar substructure in the halo and outer disk of M31. AJ 124:1452

Fisher JR, Tully RB (1976) Extensive neutral hydrogen around the peculiar spiral galaxy NGC 2146. A\&A $53: 397$

Fraternali F, Binney JJ (2006) A dynamical model for the extraplanar gas in spiral galaxies. MNRAS 366:449

Fraternali F, Binney JJ (2008) Accretion of gas onto nearby spiral galaxies. MNRAS, (in press) (astro-ph/ 0802.0496)

Fraternali F, Oosterloo T, Sancisi R, van Moorsel G (2001) A new, kinematically anomalous HI component in the spiral galaxy NGC 2403. ApJ 562:47

Fraternali F, van Moorsel G, Sancisi R, Oosterloo T (2002) Deep HI Survey of the Spiral Galaxy NGC 2403. AJ 123:3124

Fraternali F, Oosterloo T, Sancisi R, Swaters R (2005) The extra-planar neutral gas in the edge-on spiral galaxy NGC 891. In: Braun R (ed), Extra-planar Gas, Dwingeloo, ASP conf. series, vol. 331, p 239

Fukugita M, Peebles PJE (2004) The cosmic energy inventory. ApJ 616:643

García-Ruiz I, Sancisi R, Kuijken K (2002) Neutral hydrogen and optical observations of edge-on galaxies: hunting for warps. A\&A 394:769

Gentile G, Salucci P, Klein U, Granato GL (2007) NGC 3741: the dark halo profile from the most extended rotation curve. MNRAS 375:199

Giovanelli R, Haynes MP, Kent BR, Perillat P, Saintonge A, Brosch N, Catinella B, Hoffman GL, Stierwalt S, Spekkens K, Lerner MS, Masters KL, Momjian E, Rosenberg JL, Springob CM, Boselli A, Charmandaris V, Darling JK, Davies J, Lambas DG, Gavazzi G, Giovanardi C, Hardy E, Hunt LK, Iovino A, Karachentsev ID, Karachentseva VE, Koopmann RA, Marinoni C, Minchin R, Muller E, Putman M, Pantoja C, Salzer JJ, Scodeggio M, Skillman E, Solanes JM, Valotto C, van Driel W, van Zee L (2005) The Arecibo Legacy Fast ALFA Survey. I Science Goals, Survey Design, and Strategy. AJ 130:2598

Giovanelli R, Haynes MP, Kent BR, Saintonge A, Stierwalt S, Altaf A, Balonek T, Brosch N, Brown S, Catinella B, Furniss A, Goldstein J, Hoffman GL, Koopmann RA, Kornreich DA, Mahmood B, Martin AM, Masters KL, Mitschang A, Momjian E, Nair PH, Rosenberg JL, Walsh B (2007) The 
Arecibo Legacy Fast ALFA Survey. III. HI Source Catalog of the Northern Virgo Cluster Region. AJ $133: 2569$

Haynes MP (2007) HI Cosmology in the Local Universe with ALFALFA. In: Bridle A, Condon J, Hunt G (eds), Frontiers of astrophysics, ASP conference series, 18-21 June 2007, (astro-ph/0708.2547)

Haynes MP, Hogg DE, Maddalena RJ, Roberts MS, Van Zee L (1998) Asymmetry in high-precision global HI profiles of isolated spiral galaxies. AJ 115:62

Heald G, Oosterloo T (2008) Anomalous HI Gas in NGC 4395: signs of gas accretion. In: Funes JGSJ, Corsini EM (eds). Formation and evolution of galaxy disks. Rome, 1-5 October 2007 (astro-ph/ 0712.1184)

Heald GH, Rand RJ, Benjamin RA, Bershady MA (2006) Integral field unit observations of NGC 891: kinematics of the diffuse ionized gas halo. ApJ 647:1018

Heckman TM, Sancisi R, Sullivan III WT, Balick B (1982) High-resolution mapping of the giant HI envelope of the Seyfert galaxy MKN 348. MNRAS 199:425

Helmi A, de Zeeuw PT (2000) Mapping the substructure in the galactic halo with the next generation of astrometric satellites. MNRAS 319:657

Helmi A, White SDM (2001) Simple dynamical models of the Sagittarius dwarf galaxy. MNRAS 323:529

Hibbard JE, van Gorkom JH, Rupen MP, Schiminovich D (2001) An HI Rogues Gallery. In: Hibbard JE, van Gorkom JH, Rupen MP (eds). Gas and galaxy evolution, ASP conference series, vol 240, p 657

Ibata RA, Gilmore G, Irwin MJ (1994) A dwarf satellite galaxy in Sagittarius. Nature 370:194

Ibata R, Irwin M, Lewis G, Ferguson AMN, Tanvir N (2001) A giant stream of metal-rich stars in the halo of the galaxy M31. Nature 412:49

Irwin JA (1994) Arcs and bridges in the interacting galaxies NGC 5775/NGC 5774. ApJ 429:618

Irwin JA, Chaves T (2003) High-latitude HI in NGC 2613: Buoyant disk-halo outflow. ApJ 585:268

Jiang I-G, Binney JJ (1999) WARPS and cosmic infall. MNRAS 303:L7

Jozsa GIG (2007) Kinematic modelling of disk galaxies. II. A case-study of symmetrically warped galaxy disks. A\&A 468:903

Kamphuis JJ (1993) Ph.D. thesis. University of Groningen

Kamphuis J, Briggs F (1992) Kinematics of the extended HI disk of NGC 628-High velocity gas and deviations from circular rotation. A\&A 253:335

Kamphuis J, Sancisi R (1993) Widespread high velocity gas in the spiral galaxy NGC 6946. A\&A 273:L31

Kaufmann T, Mayer L, Wadsley J, Stadel J, Moore B (2006) Cooling flows within galactic haloes: the kinematics and properties of infalling multiphase gas. MNRAS 370:1612

Kereš D, Katz N, Weinberg DH, Davé R (2005) How do galaxies get their gas?. MNRAS 363:2

Kornreich DA, Haynes MP, Lovelace RVE (1998) A photometric method for quantifying asymmetries in disk galaxies. AJ 116:2154

Kornreich DA, Lovelace RVE, Haynes MP (2002) "Sloshing” librations in Lopsided disk galaxies. ApJ 580:705

Kovač K (2007) Detection of polarization in the cosmic microwave background using DASI. Ph.D. thesis, University of Groningen

Kregel M, Sancisi R (2001) NGC 3310, a galaxy merger? A\&A 376:59

Krumm N, Burstein D (1984) The extended hydrogen envelope around the dwarf galaxy DDO 154. AJ 89:1319

Lacey C, Cole S (1993) Merger rates in hierarchical models of galaxy formation. MNRAS 262:627

Larson RB (1972) Cosmogeny-Effect of infalling matter on galactic heavy element content. Nature 236:21L

Larson RB, Tinsley BM, Caldwell CN (1980) The evolution of disk galaxies and the origin of S0 galaxies. ApJ 237:692

Lee S-W, Irwin JA, Dettmar R-J, Cunningham CT, Golla G, Wang QD (2001) NGC 5775: anatomy of a disk-halo interface. A\&A 377:759

Malin D, Hadley B (1997) HI in shell galaxies and other merger remnants. PASA 14:52

Mathewson DS, Cleary MN, Murray JD (1974) The Magellanic stream. ApJ 190:291

Matteucci F (2003) The chemical evolution of the galaxy. In: McWilliam A, Rauch M (eds). Origin and evolution of the elements. Cambridge University Press, Cambridge (astro-ph/0306034)

Matthews LD, Wood K (2003) High-latitude HI in the low surface brightness galaxy UGC 7321. ApJ 593:721

Matthews LD, Uson JM (2008) HI imaging observations of superthin galaxies. II. Ic 2233 and the Blue Compact Dwarf NGC 2537. AJ 135:291

McConnachie AW, Irwin MJ, Ibata RA, Ferguson AMN, Lewis GF, Tanvir N (2003) The three-dimensional structure of the giant stellar stream in Andromeda. MNRAS 343:1335 
McNamara BR, Sancisi R, Henning PA, Junor W (1994) A violent interaction between the dwarf galaxy UGC 7636 and the giant elliptical galaxy NGC 4472. AJ 108:844

Meurer GR, Carignan C, Beaulieu SF, Freeman KC (1996) NGC 2915.II.A Dark Spiral Galaxy With a Blue Compact Dwarf Core. AJ 111:1551

Morganti R, de Zeeuw PT, Oosterloo TA, McDermid RM, Krajnović D, Cappellari M, Kenn F, Weijmans A, Sarzi M (2006) Neutral hydrogen in nearby elliptical and lenticular galaxies: the continuing formation of early-type galaxies. MNRAS 371:157

Mulder PS, van Driel W, Braine J (1995) Distribution and motions of HI and H2 in the peculiar spiral galaxy NGC 3310. A\&A 300:687

Oosterloo T (2004) High-velocity HI gas in external galaxies. In: van Woerden H, Wakker BP, Schwarz UJ, de Boer KS (eds). High-velocity clouds, astrophysics and space science library, vol 312, p 125

Oosterloo T, Fraternali F, Sancisi R (2007a) The cold gaseous halo of NGC 891. AJ 134:1019

Oosterloo TA, Morganti R, Sadler EM, van der Hulst T, Serra P (2007b) Extended, regular HI structures around early-type galaxies. A\&A 465:787

Ostriker EC, Binney JJ (1989) Warped and tilted galactic discs. MNRAS 237:785

Peek JEG, Putman ME, Sommer-Larsen J (2007) Ongoing galactic accretion: simulations and observations of condensed gas in hot halos. ApJ 674:227

Phookun B, Mundy LG, Teuben PJ, Wainscoat RJ (1992) NGC 4027-an interacting one-armed spiral galaxy with a warped ring. ApJ 400:516

Pisano DJ, Wilcots EM, Elmegreen BG (1998) The HI distribution and dynamics in two late-type barred spiral galaxies: NGC 925 and NGC 1744. AJ 115:975

Pisano DJ, Wilcots EM, Elmegreen BG (2000) Structure and star formation in NGC 925. AJ 120:763

Pisano DJ, Barnes DG, Gibson BK, Staveley-Smith L, Freeman KC, Kilborn VA (2004) Where are the high-velocity clouds in local group analogs? ApJ 610:L17

Pisano DJ, Barnes DG, Gibson BK, Staveley-Smith L, Freeman KC, Kilborn VA (2007) An HI survey of six local group analogs. I. Survey description and the search for high-velocity clouds. ApJ 662:959

Prochaska JX, Herbert-Fort S, Wolfe AM (2005) The SDSS damped ly survey: data release 3. ApJ 635:123

Rand RJ (1994) Atomic hydrogen in the NGC 4631 group of galaxies. A\&A 285:833

Reshetnikov V, Combes F (1999) Spiral galaxies with large optical WARPS. A\&AS 138:101

Richter O-G, Sancisi R (1994) Asymmetries in disk galaxies. How often? How strong? A\&A 290:L9

Rix H-W, Zaritsky D (1995) Nonaxisymmetric structures in the Stellar disks of galaxies. ApJ 447:82

Roberts M, Hogg DE, Schulman E (2001) The isolated, asymmetric spiral galaxy NGC 1637. In: Hibbard JE, Rupen M, van Gorkom JH (eds). Gas and galaxy evolution, ASP Conference Series, vol 240, p 294

Rogstad DH, Shostak GS, Rots AH (1973) Aperture synthesis study of neutral hydrogen in the galaxies NGC 6946 and IC 342. A\&A 22:111

Rots AH, Bosma A, van der Hulst JM, Athanassoula E, Crane PC (1990) High-resolution HI observations of the Whirlpool Galaxy M51. AJ 100:387

Rupen MP (1991) Neutral hydrogen observations of NGC 4565 and NGC 891. AJ 102:48

Sadler EM, Oosterloo T, Morganti R (2001) HI gas disks in elliptical galaxies. In: Funes JG, Corsini EM (eds). Galaxy disks and disk galaxies. ASP conference series, vol 230, p 285

Sadler EM, Oosterloo T, Morganti R (2002) HI in elliptical galaxies. In: Da Costa GS, Jerjen H (eds). The dynamics, structure and history of galaxies, ASP conference series, vol 273, p 215

Saglia RP, Sancisi R (1988) HI observations of the massive spiral galaxy NGC 5635. A\&A 203:28

Saha K, Combes F, Jog CJ (2007) Global lopsided instability in a purely stellar galactic disc. MNRAS 382:419

Sancisi R (1976) Warped HI Disks in Galaxies. A\&A 53:159

Sancisi R, Allen RJ (1979) Neutral hydrogen observations of the edge-on disk galaxy NGC 891. A\&A $74: 73$

Sancisi R, van Woerden H, Davies RD, Hart L (1984) Neutral hydrogen associated with the S0 galaxy NGC 1023. MNRAS 210:497

Sancisi R (1992) Gas around galaxies. Episodic infall? In: Xuan Thuan T, Balkowski C, Tran Thanh Van J (eds). Physics of nearby galaxies: nature or nurture? Proceedings of the 27th Rencontre de Moriond, Les Arcs, France, March 15-22, Gif-sur-Yvette: Editions Frontieres, p 31

Sancisi R (1999a) Galaxy interactions: the HI signature. In: Barnes JE, Sanders DB (eds). IAU Symposum 186: galaxy interactions at low and high redshift, $\mathrm{p} 71$

Sancisi R (1999b) Neutral hydrogen and dark matter in spiral galaxies. Ap\&SS 269:59 
Schaap WE, Sancisi R, Swaters RA (2000) The vertical extent and kinematics of the HI in NGC 2403. A\&A 356:49L

Schiminovich D, van Gorkom JH, van der Hulst JM, Kasov S (1994) Discovery of neutral hydrogen associated with the diffuse shells of NGC 5128 (Centaurus A). ApJ 423:L101

Schiminovich D, van Gorkom JH, van der Hulst JM, Malin DF (1995) Neutral hydrogen associated with shells and other fine structure in NGC 2865: A dynamically young elliptical? ApJ 444:L77

Schiminovich D, van Gorkom J, van der Hulst T, Oosterloo T, Wilkinson A (1997) Imaging and kinematics of neutral hydrogen in and around "Shell Galaxies". The nature of elliptical galaxies, 2nd Stromlo Symposium, vol 116, p 362

Shang Z, Brinks E, Zheng Z, Chen J, Burstein D, Su H, Byun Y-I, Deng L, Deng Z, Fan X, Jiang Z, Li Y, Lin W, Ma F, Sun W-H, Wills B, Windhorst RA, Wu H, Xia X, Xu W, Xue S, Yan H, Zhou X, Zhu J, Zou Z (1998) Ring structure and warp of NGC 5907: interaction with dwarf galaxies. ApJ 504:23L

Shapiro PR, Field GB (1976) Consequences of a new hot component of the interstellar medium. ApJ 205:762

Shostak GS, Hummel E, Shaver PA, van der Hulst JM, van der Kruit PC (1982) NGC 1961—stripping of a supermassive spiral galaxy. A\&A 115:293

Simkin SM, van Gorkom JH, Hibbard JE, Su H-J (1987) Markarian 348 - a tidally disturbed Seyfert galaxy. Science 235:1367

Sommer-Larsen J (2006) Where Are the "Missing" Galactic Baryons? ApJ 644:1

Smith BJ (1994) Optical imaging and high spatial resolution 21 CM HI observations of the peculiar galaxy NGC 2782 (Arp 215). AJ 107:1695

Swaters RA, Sancisi R, van der Hulst JM (1997) The H i Halo of NGC 891. ApJ 491:140

Swaters RA, Schoenmakers RHM, Sancisi R, van Albada TS (1999) Kinematically lopsided spiral galaxies. MNRAS 304:330

Tacconi LJ, Young JS (1986) The distribution of interstellar matter in the Scd galaxy NGC 6946. I-the neutral hydrogen disk. ApJ 308:600

Taramopoulos A, Payne H, Briggs FH (2001) HI observations of the starburst galaxy NGC 2146. A\&A $365: 360$

Thilker DA, Braun R, Walterbos RAM, Corbelli E, Lockman FJ, Murphy E, Maddalena R (2004) On the continuing formation of the andromeda galaxy: detection of $\mathrm{HI}$ clouds in the M31 halo. ApJ 601:39L

Thilker DA, Bianchi L, Meurer G, Gil de Paz A, Boissier S, Madore BF, Boselli A, Ferguson AMN, Muoz-Mateos JC, Madsen GJ, Hameed S, Overzier RA, Forster K, Friedman PG, Martin DC, Morrissey P, Neff SG, Schiminovich D, Seibert M, Small T, Wyder TK, Donas J, Heckman TM, Lee Y-W, Milliard B, Rich RM, Szalay AS, Welsh BY, Yi SK (2007) A search for extended ultraviolet disk (XUV-Disk) galaxies in the local universe. ApJS 173:538

Tinsley BM (1980) Evolution of the stars and gas in galaxies. In: Fundamentals of cosmic physics, vol 5, pp 287-388

Tinsley BM (1981) Chemical evolution in the solar neighborhood. IV-Some revised general equations and a specific model. ApJ 250:758

Tosi M (1988) Models of galactic chemical evolution-The problem of uniqueness. A\&A 197:33

Twarog BA (1980) The chemical evolution of the solar neighborhood. II-The age-metallicity relation and the history of star formation in the galactic dis. ApJ 242:242

van der Hulst JM, Sancisi R (1988) High-velocity gas in M101. AJ 95:1354

van der Hulst JM, van Albada TS, Sancisi R (2001) The Westerbork HI Survey of Irregular and Spiral Galaxies, WHISP. In: Hibbard JE, Rupen M, van Gorkom JH (eds). Gas and galaxy evolution. ASP Conference series, vol 240, p 451

van der Hulst JM, Sancisi R (2005) Gas accretion in galactic disks. In: Braun R (ed). Extra-planar gas, ASP conference series, vol 331, p 139

van Gorkom JH, Schiminovich D (1997) HI in elliptical galaxies. In: Arnaboldi M, Da Costa GS, Saha P (eds). The nature of elliptical galaxies, ASP conference series, vol 116, p 310

van Woerden H, Wakker BP (2004) Distances and Metallicities of Hvcs. In: van Woerden H, Wakker BP, Schwarz UJ, de Boer KS (eds). High-velocity clouds. Astrophys Space Sci Lib 312:195

van Woerden H, Wakker BP, Schwarz UJ, de Boer KS (2004) High-Velocity Clouds. In: van Woerden H, Wakker BP, Schwarz UJ, de Boer KS (eds). Astrophys Space Sci. Lib. 312, Kluwer, Dordrecht

Verheijen MAW, Sancisi R (2001) The Ursa Major cluster of galaxies. IV. HI synthesis observations. A\&A 370:765

Wakker BP, van Woerden H (1997) High-Velocity Clouds. ARA\&A 35:217 
Wakker BP, York DG, Howk C, Barentine JC, Wilhelm R, Peletier RF, van Woerden H, Beers TC, Ivezic Z, Richter P, Schwarz UJ (2007) Distances to galactic High-Velocity Clouds: complex C. ApJ 670:113L

Wakker BP, York DG, Wilhelm R, Barentine JC, Richter P, Beers TC, Ivezic Z, Howk JC (2008) Distances to galactic High-Velocity Clouds. I. Cohen stream, Complex GCP, Cloud g1. ApJ 672:298

White SDM, Frenk CS (1991) Galaxy formation through hierarchical clustering. ApJ 379:52

Yun MS, Ho PTP, Lo KY (1994) A high-resolution image of atomic Hydrogen in the M81 Group of Galaxies. Nature 372:530

Zaritsky D, Rix H (1997) Lopsided spiral galaxies and a limit on the galaxy accretion rate. ApJ 477:118

Zwaan MA, Briggs FH, Sprayberry D (2001) The luminosity function and surface brightness distribution of HI selected galaxies. MNRAS 327:1249

Zwaan MA, Meyer MJ, Staveley-Smith L, Webster RL (2005) The HIPASS catalogue: $\Omega_{\mathrm{HI}}$ and environmental effects on the HI mass function of galaxies. MNRAS 359:30L

Zwaan MA, van der Hulst JM, Briggs FH, Verheijen MAW, Ryan-Weber EV (2005) Reconciling the local galaxy population with damped Lyman cross-sections and metal abundances. MNRAS 364:1467 